\title{
Du fleuve au canal : enquête sur le patrimoine fluvial du Blavet (Bretagne) du Moyen Âge à nos jours
}

From the river to the canal: investigation of the fluvial heritage of the Blavet river (Brittany) from the Middle Ages to present day

Vom Fluss zum Kanal: Untersuchung zum kulturgeschichtlichen Erbe des Flusses Blavet (Bretagne)

Del río al canal: investigaciones sobre el patrimonio fluvial del río Blavet

(Bretaña) desde la Edad Media hasta nuestros días

\section{Pauline Peter}

\section{OpenEdition}

Journals

Édition électronique

URL : https://journals.openedition.org/rao/6558

DOI : $10.4000 /$ rao.6558

ISSN : 1775-3732

Éditeur

Presses universitaires de Rennes

Édition imprimée

Date de publication : 21 décembre 2021

Pagination : 275-299

ISBN : 978-2-7535-8668-0

ISSN : 0767-709X

\section{Référence électronique}

Pauline Peter, « Du fleuve au canal : enquête sur le patrimoine fluvial du Blavet (Bretagne) du

Moyen Âge à nos jours », Revue archéologique de l'Ouest [En ligne], 37 | 2021, mis en ligne le 21 décembre 2021, consulté le 11 octobre 2022. URL : http://journals.openedition.org/rao/6558 ; DOI : https://doi.org/10.4000/rao.6558 


\title{
Du fleuve au canal : enquête sur le patrimoine fluvial du Blavet (Bretagne) du Moyen Âge à nos jours
}

\author{
From the river to the canal: investigation of the fluvial heritage of the Blavet river (Brittany) \\ from the Middle Ages to present day
}

\author{
Pauline Peter \\ Doctorante en archéologie, université de Nantes, LARA, UMR6566 CReAAH (pauline.peter@univ-nantes.fr)
}

\begin{abstract}
Résumé : Le Blavet est un fleuve breton canalisé sur la moitié de son cours au XIxe. L'étude des archives des Ponts-et-Chaussées a mis en évidence de nombreux aménagements fluviaux liés au cours d'eau (pêcheries, moulins, passages à gué, etc.) partiellement détruits par ces travaux de grande ampleur. Cette documentation d'une qualité exceptionnelle apporte également des informations sur l'architecture de ces installations mais aussi sur les choix faits par les ingénieurs pour canaliser la rivière.
\end{abstract}

\begin{abstract}
The Blavet is an armorican river canalised for half of its course in the 19th century. The study of the civil engineering archives highlights numerous river developments linked to the watercourse (fisheries, mills, fords, etc.) destroyed by this large-scale work. This documentation of exceptional quality also provides information on the architecture of these installations but also on the choices made by engineers to canalise the river.
\end{abstract}

Mots clés: Bretagne, Blavet, archives, patrimoine fluvial, Moyen Âge.

Keywords: Bretagne, Blavet, archives, river heritage, Middle Ages.

Ces dernières décennies, l'archéologie fluviale est devenue un champ de recherche nécessaire à la compréhension des territoires et des relations entre l'homme et son milieu. Des travaux précurseurs d'É. Rieth sur la Charente ou encore de L. Bonnamour sur la Saône dans les années 1970 découlent de nombreuses études qui mettent en valeur de grands cours d'eau, comme la Loire, mais aussi des fleuves et des rivières que l'on peut qualifier de "secondaires " (Dumont, 2006; Serna, 2013). Néanmoins, les canaux suscitent un intérêt encore assez limité, alors que la canalisation et la création de nouvelles voies d'eau ont une incidence certaine sur le patrimoine archéologique fluvial et l'utilisation même de la rivière. Contrairement aux canaux antiques et médiévaux ${ }^{1}$, les grands canaux de navigation - construits à partir du XVII ${ }^{\mathrm{e}} \mathrm{s}$. - ont bénéficié d'une attention particulière de la part des historiens, qui se sont attachés essentiellement aux qualités techniques, aux ouvrages d'art ainsi qu'à la portée économique de ces grands aménagements. Créés dans le

1. Qui font l'objet de notre thèse de doctorat depuis septembre 2017 à l'université de Nantes (dir. M. Monteil, J. Mouchard) but de développer un réseau de navigation intérieure performant et compétitif, leur construction est à l'origine de quantités d'archives, très utiles pour reconstituer le paysage archéologique de cours d'eau qui ont été profondément transformés et artificialisés. Bien que régulièrement dragués, ils ne sont pourtant que rarement soumis à un suivi archéologique, alors que certains vestiges encore conservés peuvent être approchés, notamment lors de la mise à sec des biefs pour en assurer l'entretien.

C'est dans ce cadre que s'inscrit cette étude consacrée au Blavet, initiée en 2014, au cœur d'un nouveau projet décennal de dragage prévu par la Région Bretagne, dans le but d'en maintenir la navigabilité (Peter, 2015). Fleuve côtier de près de $150 \mathrm{~km}$ de long, le Blavet traverse la péninsule armoricaine du nord au sud depuis sa source, dans les Côtes-d'Armor, jusqu'à la rade de Lorient, dans le Morbihan (fig. 1). L'absence d'un volet archéologique au sein de l'expertise préalable à ce récent projet de dragage reflète, entre autres, le manque de connaissances sur l'anthropisation du cours d'eau, canalisé sur une portion 
de son cours au début du XIX ${ }^{e}$ s. Le croisement des sources documentaires - principalement liées à l'artificialisation de la rivière - et les observations pratiquées sur le terrain apportent un nouvel éclairage sur le Blavet avant, pendant et après sa canalisation.

\section{LE BLAVET, MORPHOLOGIE D'UN FLEUVE}

Depuis sa source, au sud de Bourbriac (Côtes-d'Armor), à son estuaire (Morbihan), le Blavet traverse ou borde 35 communes sur un linéaire de près de $150 \mathrm{~km}$ et profite de l'apport en eau d'une vingtaine d'affluents, dont l'Ével et la Sarre (fig. 2). En amont, la rivière creuse un substrat de granite qui devient schisteux sur la majeure partie de son tracé. Au plus proche de son embouchure, le soussol est principalement composé de produits granitiques (Cogné et al., 1972).

Aujourd'hui, seule la portion entre Pontivy et Hennebont (Morbihan) est encore connue sous le nom de canal du Blavet. Son cours est ponctué de 28 écluses et $60 \mathrm{~km}$ séparent l'écluse des Récollets (Pontivy) de celle de Polvern (Hennebont). Pourtant, la canalisation du Blavet, telle qu'elle a été mise en œuvre à l'aube du XIX ${ }^{e}$ s., constituait un programme bien plus vaste. En effet, c'est à partir de Pontivy que le Blavet se connectait au canal de Nantes à Brest, au moyen de 32 écluses supplémentaires. Celui-ci suivait le tracé de la rivière jusqu'à Gouarec (Côtes-d'Armor) pour emprunter ensuite le ruisseau du Doré et se diriger vers Brest. Cependant, la création de l'usine hydroélectrique de Guerlédan à Saint-Aignan, entre 1923 et 1930, sonne le glas de ce système instauré moins d'un siècle plus tôt. La mise en eau du barrage a entraîné la submersion de près de $13 \mathrm{~km}$ de canal et 18 écluses sous un lac de plus de 300 ha; les autres sont alors coupées du réseau et donc désaffectées.

Le Blavet peut être divisé en quatre sections : le haut Blavet (de sa source à Bourbriac jusqu’à Gouarec), la section commune avec le canal de Nantes à Brest (de Gouarec à Pontivy), l'actuel canal du Blavet (de Pontivy à Hennebont) et enfin le Blavet maritime (d'Hennebont à l'estuaire) [fig. 3]. Chacune d'elles possède des caractéristiques propres et toutes n'ont pas été anthropisées de la même manière. De surcroît, la quantité et la qualité de la documentation archivistique sont inégales selon les sections : elle est plus importante pour la portion canalisée que pour la partie septentrionale du cours d'eau.

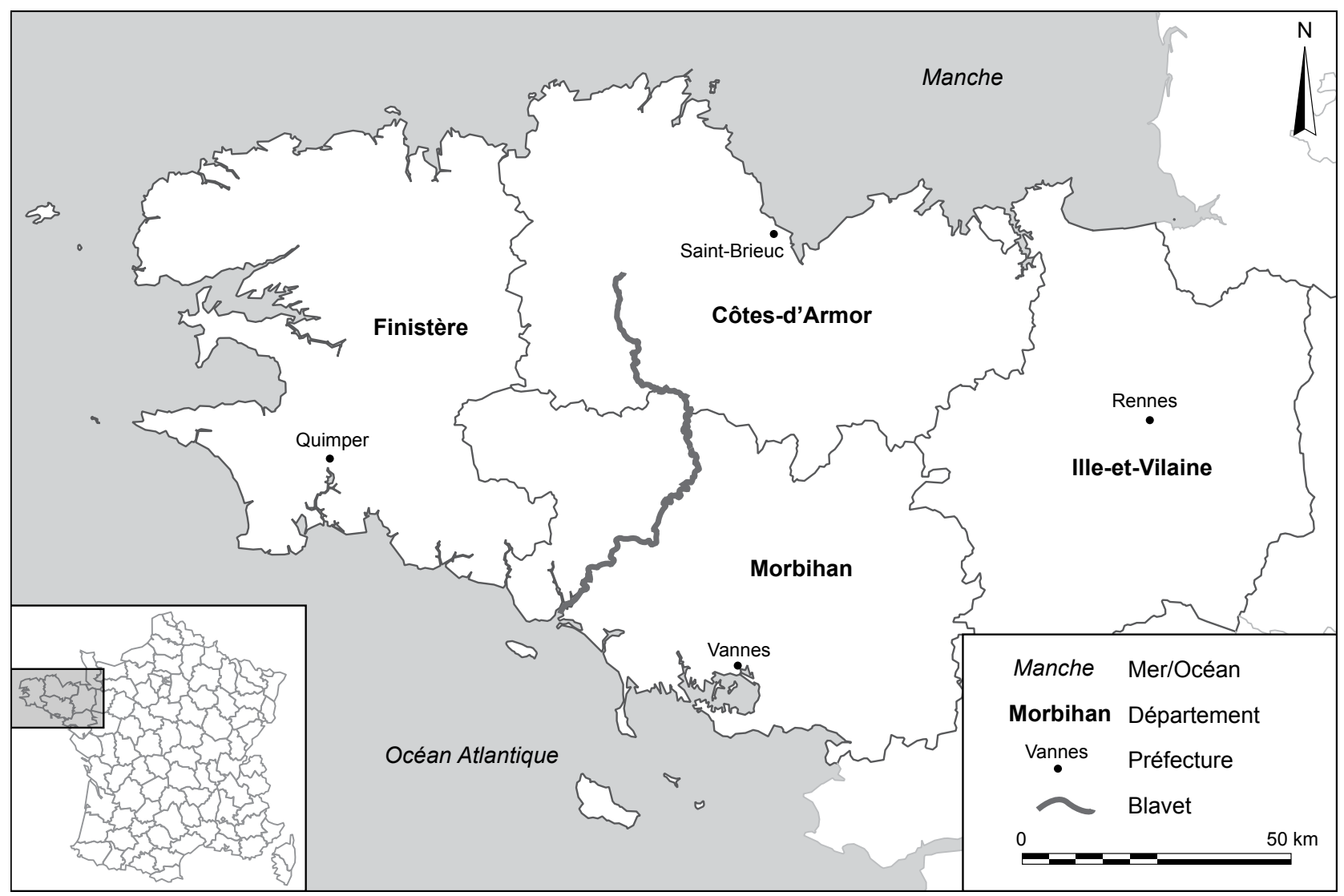

Figure 1 : Situation géographique du Blavet (réal. P. Peter).

Figure 1: Location of the Blavet river (๑ P. Peter). 


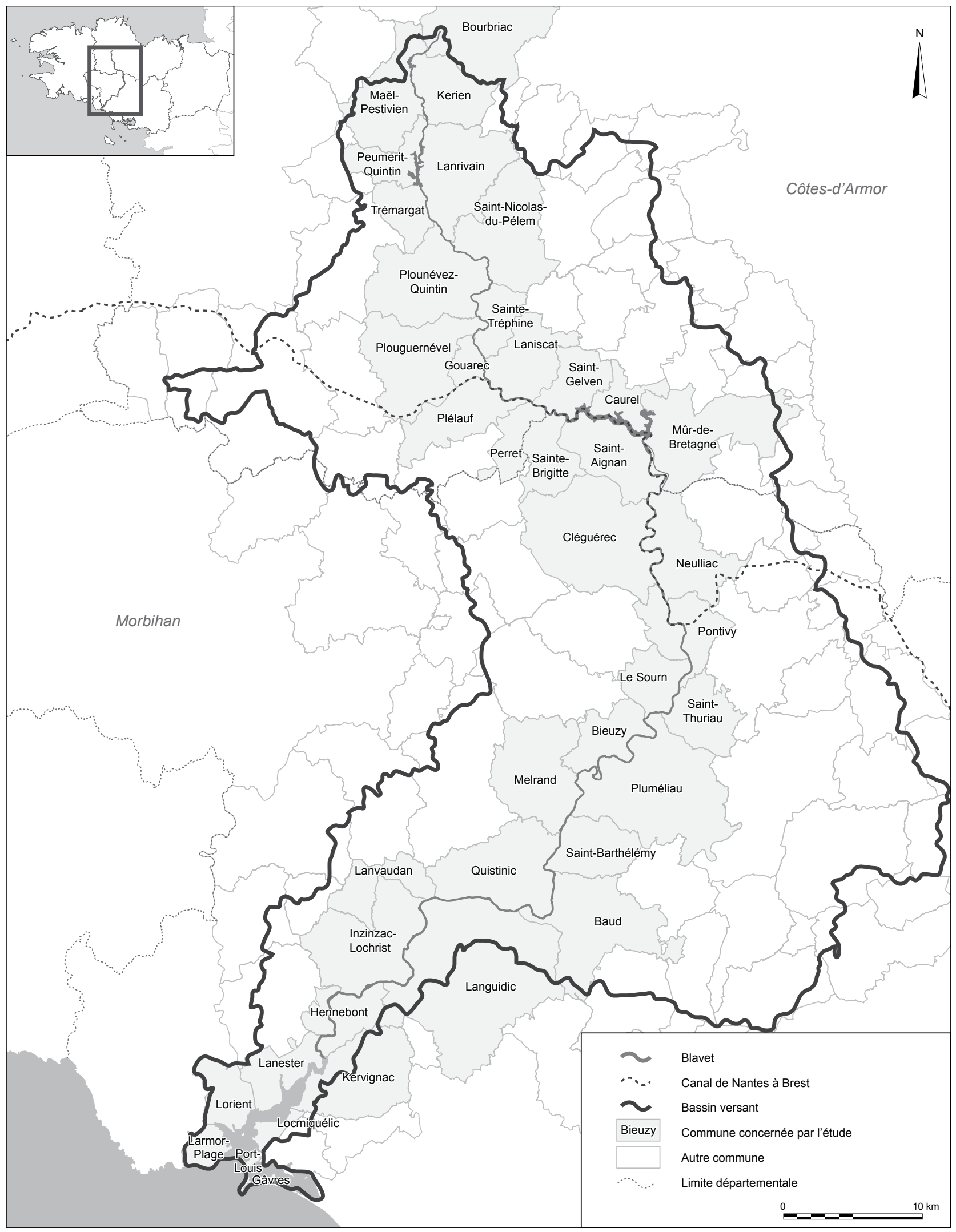

Figure 2 : Bassin versant du Blavet et communes concernées par l'étude (real. P. Peter, source : BD Carthage, SAGE Blavet).

Figure 2: The Blavet's drainage basin and the study area (O P. Peter, source: BD Carthage, SAGE Blavet). 


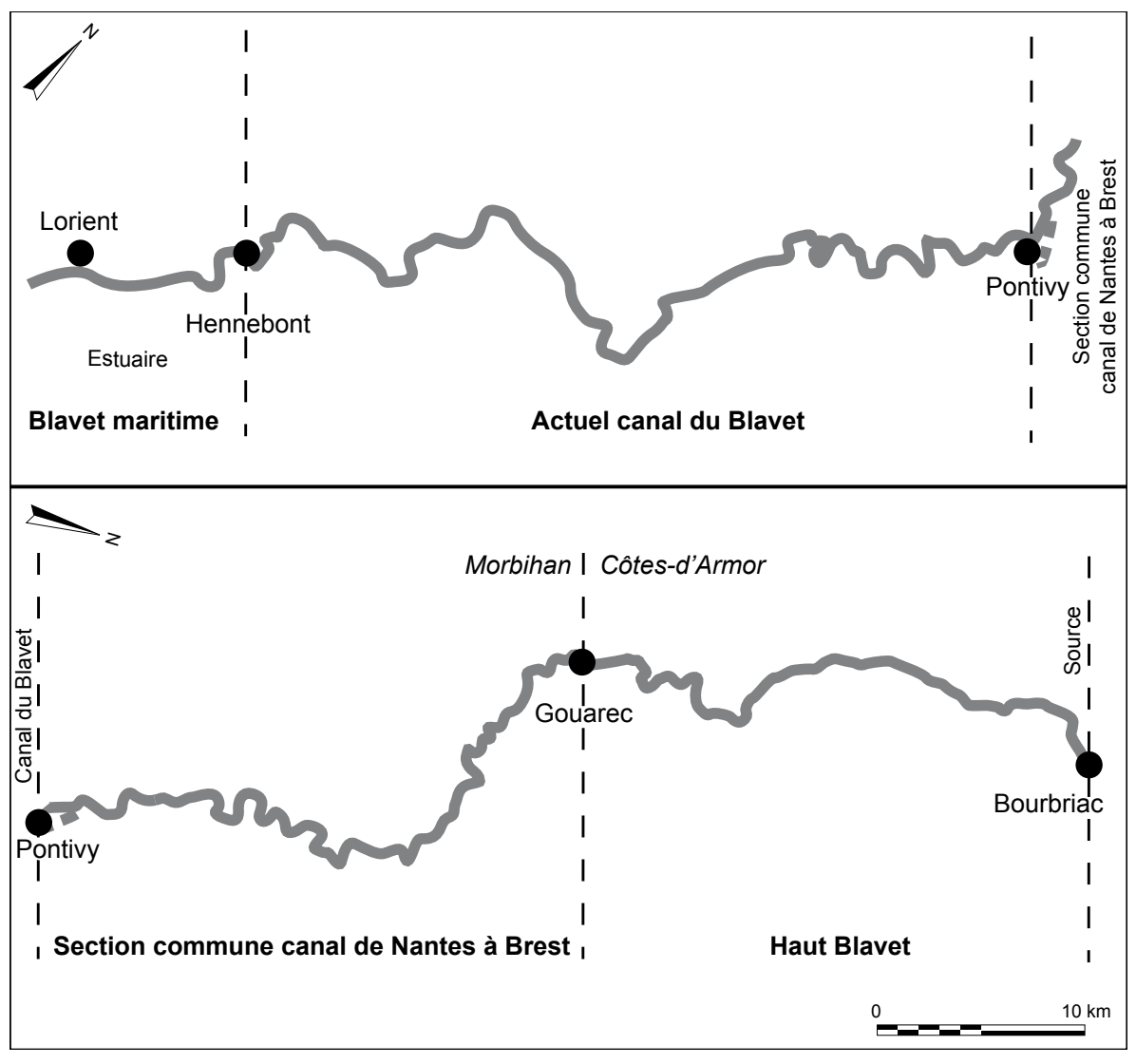

Figure 3 : Représentation schématique des grands ensembles constitutifs du Blavet (réal. P Peter).

Figure 3: Schematic drawing of the ensembles that make up the Blavet (c) P. Peter)

\section{DE LA RIVIÈRE AU CANAL : BRÈVE HISTOIRE DE LA CANALISATION DU BLAVET}

\section{Contexte historique}

Moins coûteux que le transport terrestre, le transport par voie fluviale a toujours occupé une place prépondérante dans le commerce et les échanges. Lorsque la rivière n'est pas praticable ou n'offre pas la possibilité d'atteindre un point donné, la construction d'un canal permet de pallier cette difficulté. L'inventaire des canaux de navigation métropolitains, initié depuis 2015, témoigne d'une pratique déjà répandue à l'époque romaine. À la fin du $\mathrm{XV}^{\mathrm{e}}$ s., l'arrivée de l'écluse à sas révolutionne les techniques de canalisation et donne l'impulsion à la création d'un réseau de navigation intérieure de grande envergure (fig. 4). Le canal de Briare (1604-1642), premier ouvrage à relier deux bassins versants - la Loire et la Seine -, préfigure ainsi l'artificialisation massive des rivières. Ce canal à point de partage ${ }^{2}$, construit dans le but de désenclaver Paris, s'affranchit des difficultés liées à l'alimentation en

2. Le fonctionnement du canal à point de partage est rendu possible grâce à une succession de biefs, délimités par des écluses à sas qui font monter ou descendre le niveau d'eau. Il est ainsi possible de gravir ces biefs à la manière des marches d'un escalier. Le bief situé au sommet est alimenté par une source annexe (de type lac, étang, réservoir), qui assure la présence constante d'eau au sein des différents eau (Pinon, 2005, p. 27). Terrain de toutes les expérimentations, le canal de Briare compte, par ailleurs, l'un des plus célèbres ponts-canaux de France. Il est suivi par la création du canal du Midi (1667-1687), qui a pour dessein de relier la Méditerranée à l'océan Atlantique (Pinon, 2005, p. 55). La seconde moitié du XviII ${ }^{e}$ s. est marquée par l'avènement des grands canaux (canal de Bourgogne, du Centre, du Nivernais, etc.). Connecter les différentes régions du royaume devient une réelle priorité.

L'idée de doter la Bretagne de canaux prend racine au XVIII ${ }^{\mathrm{e}}$ s., dans un contexte géopolitique profondément marqué par les rivalités qui opposent la France et l'Angleterre. En effet, les tensions entre ces deux nations pour conserver leurs colonies et leurs droits maritimes transparaissent dans la volonté d'offrir à la Bretagne un accès maritime depuis l'intérieur des terres. En raison de sa proximité avec les îles Britanniques, la Bretagne, qui subit plusieurs débarquements entre 1757 et 1761 et de nombreux blocus de ses ports, est bien trop exposée aux attaques. Elle est également le théâtre de plusieurs batailles navales, dont celle des Cardinaux (20 novembre 1759) au large de Quiberon (Chaline, 2004, p. 38, 286). Malgré la ratification de plusieurs traités, dont la paix d'Amiens en

segments du canal. Sa traversée permet ainsi de franchir la ligne de crête et donc de passer d'un bassin versant à un autre. 
Figure 4 : Fonctionnement d'une écluse à sas (réal. P. Peter).

Figure 4: The workings of a lock (C P. Peter).

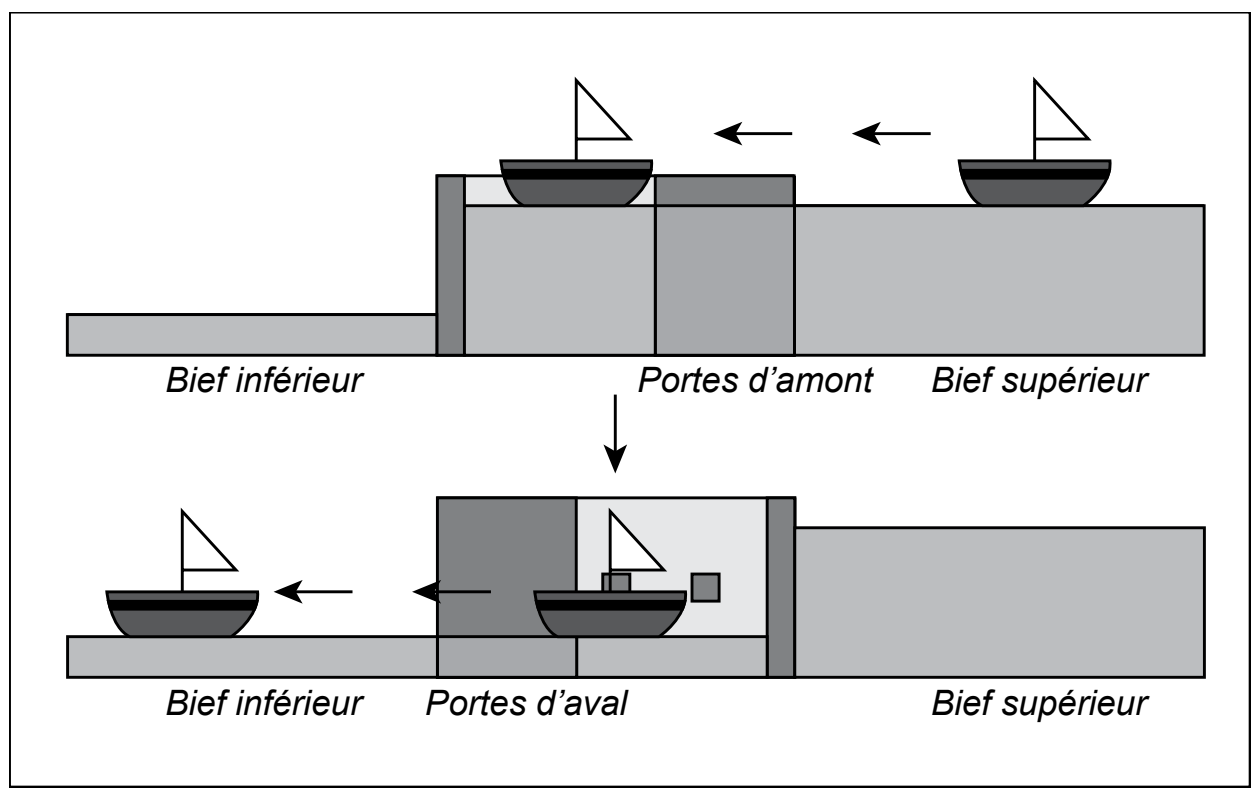

1802, de nouveaux conflits éclatent. L'arrivée au pouvoir et la soif expansionniste de Napoléon Bonaparte n'arrangent en rien les relations entre les deux pays. Un peu plus d'un an après la signature du traité, la guerre reprend avec comme point d'orgue la défaite française lors de la bataille de Trafalgar en 1805 (Boudon, 2006, p. 162163). On comprend que, dans ce climat belliqueux et pour faire face aux échecs et aux blocus, la Bretagne doive se doter d'un réseau de navigation intérieure performant afin de se protéger de la menace anglaise.

\section{Le projet de François-Joseph de Kersauson (1746 et 1765)}

Les premières propositions sérieuses de canalisation des cours d'eau bretons naissent sous la plume de F.-J. de Kersauson, dès le milieu du xviII ${ }^{\mathrm{e}} \mathrm{s}$. Les deux mémoires qu'il présente en 1746 et 1765 devant les États de Bretagne font écho aux affrontements franco-britanniques et à la nécessité d'améliorer les liaisons fluviales pour désenclaver les ports militaires. D’après lui, construire ces canaux est essentiel car ils offriront une navigation plus sûre, créeront de nouvelles voies commerciales et, surtout, assureront un accès permanent aux ports de Brest, de Saint-Malo ou encore de Lorient. Ses propos préfigurent ainsi la création des canaux de Nantes à Brest, de l'Ille-et-Rance mais aussi du Blavet. Ce dernier sera opérationnel « après qu’on aura nettoyé, et par la même opération creusé et étréci en quelques endroits » le lit du «Blaved " (Kersauson, 1886, p. 103). La lutte intestine entre la France et l'Angleterre transparaît nettement dans ses propos : ainsi « la France sera toujours la Puissance dominante de l'Europe, pendant qu'elle aura en Mer durant la paix, autant de Vaisseaux de guerre que l'Angleterre, et qu'à chaque commencement de guerre elle pourra promptement augmenter le nombre de ces Vaisseaux, et les armer tous de façon avantageuse " (Kersauson, 1886, p. 108). La dimension stratégique des canaux bretons dépasse alors le seul intérêt économique d'une telle entreprise.

\section{Décrire le Blavet avant sa canalisation : le temps des reconnaissances}

Près de soixante ans s'écoulent entre le projet de F.-J. de Kersauson et le début de la canalisation du Blavet. Quelques descriptions du fleuve apportent des informations quant à son aspect ainsi que sur les obstacles qui ponctuent son cours. Avant que ne soient entreprises les premières reconnaissances menées par des ingénieurs, un premier aperçu du Blavet est livré par un certain Chardon. Durant son périple, décrit dans une lettre adressée aux États de Bretagne en 1779, il emprunte le Blavet jusqu'à Hennebont à l'aide d'une petite barque, dont il doit descendre régulièrement pour franchir pêcheries, chaussées de moulin et hauts-fonds (arch. dép. Morbihan, S 2518). Si son récit est vivement critiqué par les ingénieurs en charge de la canalisation du Blavet, il n'en reste pas moins la plus ancienne mention illustrant l'état général de la rivière dans le dernier quart du XVIII ${ }^{\mathrm{e}} s$.

Quelques années plus tard, en 1785, l'ingénieurgéographe A. Laumailler est officiellement chargé de mener une première campagne de reconnaissance. Son mémoire, dans lequel il intègre ses observations et des prises de mesures, est très utile pour dresser un inventaire des installations fluviales - pêcheries, moulins, 
ponts, etc. - présentes sur le Blavet avant sa canalisation, de même que les obstacles naturels - rochers, hauts-fonds, îlots - qui rendent difficile la navigation (arch. dép. Morbihan, 21C 480). La Révolution française et la Chouannerie orientent les discussions sur des questions bien éloignées du réseau fluvial breton et aucune suite n'est immédiatement donnée à la reconnaissance d'A. Laumailler. Le débat sur la canalisation est relancé lorsque l'ingénieur en chef des Ponts et Chaussées P.-J.-B. Pichot et l'ingénieur ordinaire G. Bouëssel sont mandatés pour sillonner les rives du Blavet jusqu'à Hennebont, en 1802. Des plans et des profils très détaillés du cours d'eau sont alors réalisés afin de renseigner le plus précisément possible la topographie, face à l'imminence du début des travaux réclamés par Napoléon. En effet, la réalisation du canal du Blavet est ordonnée par l'État le 17 septembre 1802 et la mission de reconnaissance commence le 21 septembre suivant (Nicolas et Cheyrouze, 2015, p. 16). Les observations et les préconisations des deux ingénieurs sont consignées dans un mémoire rendu le 4 octobre 1802 : ils y répertorient des dizaines d'obstacles entravant le cours d'eau et qu'il conviendrait d'éliminer afin de le rendre navigable (arch. dép. Morbihan, 3S 373 à 375). Le texte est complété par des plans d'ensemble ou de détail dressés entre novembre 1802 et novembre 1803, sur lesquels sont figurés les futures écluses et le chemin de halage. Les obstacles, naturels ou anthropiques, sont inscrits à l'encre rouge ou noire, de même que les curages, usurpations ${ }^{3}$ et remblaiements nécessaires au bon établissement du canal (Arch. nat., F14 10052.2) [fig. 5]. Face à la quantité et surtout à la qualité de la documentation produite par G. Bouëssel et son équipe - et à la suite de la volonté de moderniser Pontivy alors rebaptisée Napoléonville - il n'y a plus de doute possible quant à la construction du canal du Blavet. Celle-ci est définitivement approuvée en mars 1804 et s'achèvera en 1838 , après une ouverture partielle en 1825(Pinon, 1986). En parallèle, sont lancés les chantiers du canal d'Ille-et-Rance (1804-1837), dans le prolongement de la Vilaine déjà canalisée sous François I ${ }^{\text {er }}$, et celui du canal de Nantes à Brest, qui vont durer jusqu'en 1842 (fig. 6).

\section{DES SOURCES VARIÉES AU SERVICE DE L'ARCHÉOLOGIE : LE COURS D'EAU RETROUVÉ}

Comprendre la canalisation du Blavet et ses conséquences sur le patrimoine archéologique lié à la rivière

3. Ce terme est employé dans les documents pour désigner l'élargissement ponctuel de la rivière. L'apport de sédiments pour en réduire la largeur est qualifié, au contraire, de remblaiement. Ces opérations ont pour but de régulariser le tracé du cours d'eau.

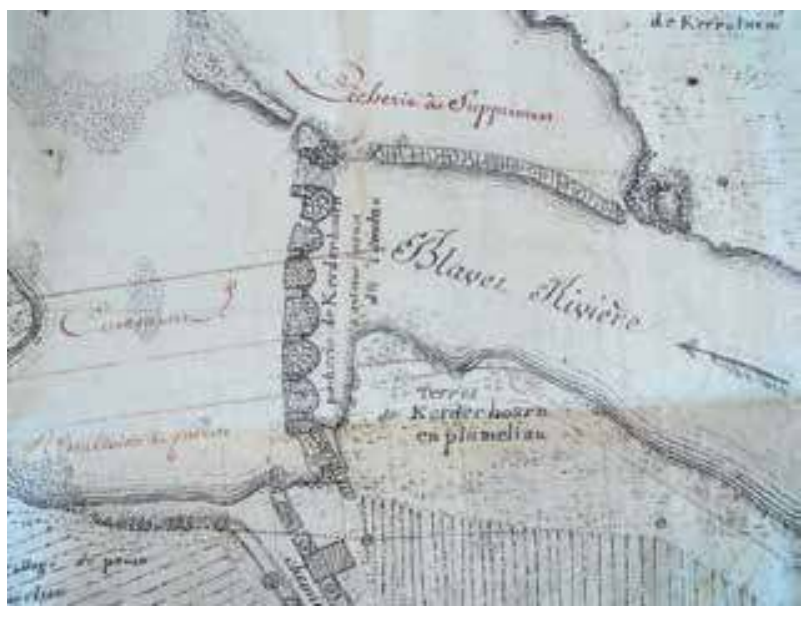

Figure 5 : Extrait du « plan de la pêcherie de Kerderhoarn en Pluméliau avec ses abords, élevé en Floréal an XI $॥$, avril-mai 1803. Les annotations en rouge datent de 1804 (cliché P. Peter, source : arch. dép. Morbihan, 3 S 374).

Figure 5: Extract from the Kerderhoarn en Pluméliau fishery and its surrounding area, built in "Floréal an XI", April-May 1803. The red notes date from 1804 (photo: P. Peter, source: arch. dép. Morbihan, 3S 374).

a nécessité le croisement de sources variées et de faire appel à plusieurs champs disciplinaires. Depuis près d'un demi-siècle, l'intérêt grandissant des historiens et historiens des techniques pour les canaux a donné naissance à plusieurs monographies et ouvrages de synthèse (Pinon, 1986). Les canaux bretons ne sont pas en reste, mais le Blavet y est souvent cité comme une composante du canal de Nantes à Brest et non pas comme une entité à part entière (Benferhat et al., 1999, par exemple). Rares sont les études qui s'attachent aux cours d'eau avant leur canalisation car c'est principalement ce phénomène qui attire l'attention. De plus, les recherches ont rapidement été limitées par le manque d'informations, lié à la lente intégration du patrimoine fluvial au sein de la Carte archéologique nationale, due à l'absence de prospections systématiques. Dans la base Patriarche, seules deux entités étaient alors référencées sur le Blavet. La première correspond au moulin de Rimaison à Bieuzy (Morbihan), inscrit sur la liste des Monuments historiques, et l'autre au pont d'Hennebont.

Il a donc fallu adopter une démarche régressive et consulter les textes et plans établis dans le cadre de la construction du canal, conservés aux archives départementales du Morbihan (Vannes), des Côtes-d'Armor (Saint-Brieuc) et aux Archives nationales (site de Pierrefitte-sur-Seine). Ainsi, les archives des Ponts et Chaussées (arch. dép. Morbihan, série $S$; arch. dép. Côtes-d'Armor, série 1S), de la navigation intérieure (arch. dép. Morbihan, série 3S), du service hydraulique (arch. dép. Morbihan, série 7S) et des travaux publics (Arch. nat., série F14) ont été dépouillées. La richesse de la documentation et surtout l'excellente qualité des plans 


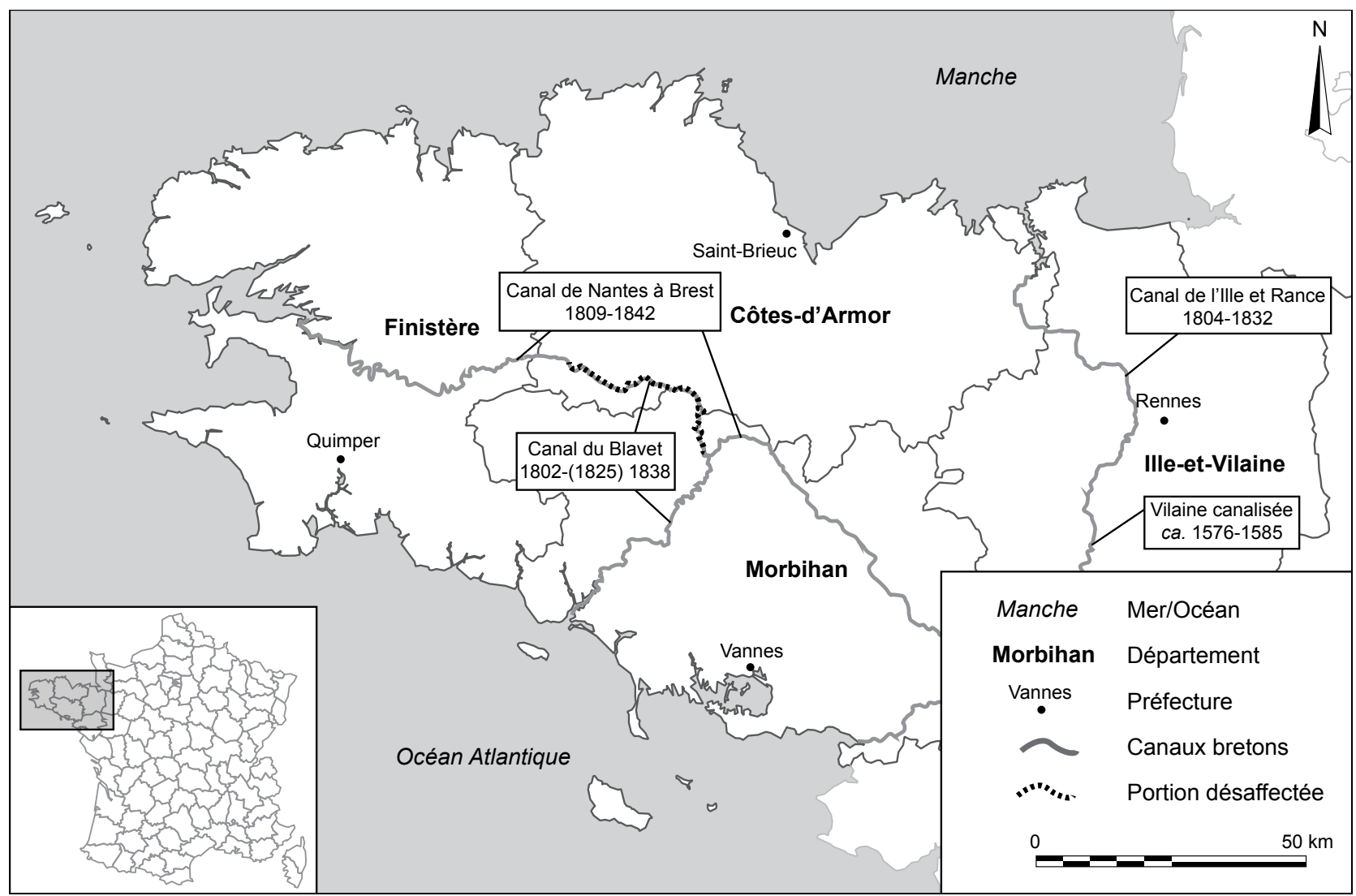

Figure 6 : Cartographie des canaux bretons (réal. P. Peter, source : BD Carthage).

Figure 6: Map of the Brittany canals (C P. Peter, source: BD Carthage).

ont permis d'identifier et de localiser avec une grande précision les aménagements présents sur le Blavet avant sa canalisation. La superposition de plusieurs éléments de nature cartographique - cadastre napoléonien, carte d'état-major, carte de Cassini, photographies aériennes, etc. - a également livré de bons résultats. Outre ces documents techniques, les archives de l'abbaye Notre-Dame de la Joie à Hennebont ont été très utiles pour évaluer la pérennité - ou non - des pêcheries possédées par les religieuses (arch. dép. Morbihan, H 60). Les litiges liés à l'utilisation même de ces pièges à poissons, souvent partagés entre plusieurs propriétaires riverains, sont par ailleurs à l'origine de notes et de procès-verbaux qui témoignent de l'existence, de l'entretien voire de la destruction de ces installations.

En complément, les observations réalisées en 2013 et 2014 par Jean-François Nicolas (Groupe d'Histoire et Patrimoine de Baud), lors de la mise à sec des biefs du canal ont été très instructives. En effet, certains aménagements sont encore conservés au sein de la rivière, comme des chaussées de moulins ou encore des radiers de pêcheries. De plus, les recherches qu'il a menées en archives ont largement contribué à ce travail et ont fait l'objet de deux publications (Nicolas et Cheyrouze, 2015, 2019).

\section{LES FORMES D'ANTHROPISATION DU BLAVET}

\section{Un riche patrimoine fluvial}

L'intérêt d'étudier un canal contemporain est de pouvoir suivre, grâce aux documents d'archives, l'ensemble de la construction, mais également les choix faits par les ingénieurs afin de réaliser une canalisation à l'économie, en tenant compte de la configuration du terrain et des ouvrages préexistants. Grâce à la démarche régressive mise en place et à la confrontation des sources mentionnées plus haut, ce sont près de deux cents aménagements liés au cours d'eau qui ont été référencés : pêcheries, moulins, sites traversiers, sans oublier les écluses, qui tous témoignent d'une anthropisation intense du cours d'eau.

\section{Les pêcheries}

Les pêcheries sont les sites les plus répandus sur le Blavet. Le fleuve est connu depuis le Moyen Âge pour ses ressources piscicoles, notamment le saumon qui en fait la renommée (Nicolas et Cheyrouze, 2015, p. 53). Les archives, et plus particulièrement les plans dressés à l'aube $\mathrm{du} \mathrm{XIX}^{\mathrm{e}}$ s., apportent des informations relatives à l'archi- 
tecture de ces installations pouvant compter jusqu'à douze pertuis $^{4}$. Il faut toutefois rester prudent quant à l'image que ces documents renvoient : il ne s'agit que du reflet du cours d'eau à un instant donné et ils n’apportent que très rarement des précisions d'ordre chronologique. De surcroît, les installations légères, non maçonnées, sont plus difficiles à percevoir car elles ne sont pas représentées sur les plans. Leur existence est pourtant mentionnée dans quelques textes d'archives car ces pièges mobiles, facilement démontables, donnent lieu à des litiges.

Les recherches en archives et sur le terrain ont néanmoins conduit à l'identification de 40 pêcheries fixes sur le cours d'eau (33 sur le canal actuel et 7 sur la section commune avec le canal de Nantes à Brest), auxquelles s'ajoutent 8 ouvrages qu'il n'a malheureusement pas été possible de localiser (fig. 7). Dans l'estuaire, ce sont 22 aménagements - dont 18 détruits - qui ont été référencés (Langouët et al., 2008, p. 124). Ces pêcheries sont essentiellement datées de l'époque moderne, même si quelques établissements médiévaux sont à signaler. Aucune pêcherie romaine n'a, en revanche, été répertoriée, mais d'autres pratiques halieutiques - plus difficiles à percevoir - sont suggérées par la découverte d'un atelier de salaisons ${ }^{5}$ à Lanester (Morbihan), à proximité duquel ont été mis au jour des lests de filet (André, 1992).

\section{Les pêcheries médiévales}

La plus ancienne mention d'une activité de pêche sur le Blavet remonte à 1125 et concerne le secteur de Bieuzy (Morbihan). En 1184, le vicomte Alain de Rohan octroie à l'abbaye de Bon Repos, qu'il fonde à Saint-Gelven, le droit d'établir pêcheries et moulins sur ses terres (Nicolas et Cheyrouze, 2015, p. 23). Les témoignages se font plus nombreux à partir du XIII ${ }^{e}$ s. Le duc de Bretagne Jean I ${ }^{\text {er }}$, autorise l'abbaye Notre-Dame de la Joie $\left(\right.$ Hennebont $\left.{ }^{6}\right)$, fondée en 1276, à construire un moulin et des pêcheries pour subvenir à ses besoins (arch. dép. Morbihan, $60 \mathrm{H}$ 27). Ce droit de pêche est difficilement respecté par les habitants qui n'hésitent pas à poser illégalement des filets dans la rivière (arch. dép. Morbihan, 60H 18). Les interdictions et les procès n'empêchent pas la mise en place de ces barrages mobiles. Au début du XIv s. les pêcheries possédées par l'abbaye sont localisées " audessus du pont de Saint-Christ ", soit Lochrist. D'autres

4. Le pertuis correspond ici à l'espace compris entre deux piles ou deux murets en pierre au sein duquel est installé le piège (nasse, filet) destiné à capturer les poissons. Lorsque ces derniers sont relevés, l'eau s'écoule par ces pertuis. Certains d'entre eux sont par ailleurs réservés à la circulation des embarcations. 5. Un établissement semblable a été fouillé à une vingtaine de kilomètres au sud-est à Étel (Driard et al., 2017).

6. L'important fonds d'archives de l'abbaye Notre-Dame de la Joie a été légué aux archives départementales du Morbihan, à Vannes (arch. dép. Morbihan, $60 \mathrm{H}$ ). ouvrages (pêcheries du Poulgui, du Guerne, du Garu, de Bily) complètent leurs possessions et sont régulièrement mentionnées dans les archives (arch. dép. Morbihan, $60 \mathrm{H} 21$ et 27). Au total, six à huit pêcheries ont été identifiées à proximité d'Hennebont, mais seules deux d'entre elles - les pêcheries des ponts de Lochrist - ont été réellement localisées. Au regard des informations recueillies à travers la consultation d'une infime portion de la documentation, le fonds d'archives de l'abbaye de la Joie mériterait une étude plus approfondie de l'ensemble de ses dossiers, de même que celui de l'abbaye de Bon Repos à Saint-Gelven, conservé aux archives départementales des Côtes-d'Armor.

\section{Les pêcheries à l'époque moderne}

Plusieurs textes datés du XVII ${ }^{\mathrm{e}}$ s. permettent de cerner les activités halieutiques. Ce sont principalement des litiges qui opposent les abbesses de la Joie aux seigneuries voisines. De nouveaux ouvrages (pêcherie du Garff, du bourg de Lochrist, du Grand Guildo, etc.) apparaissent dans les archives (arch. dép. Morbihan, 60H 21 et 44). Une fois encore, les informations sont trop lacunaires pour localiser ces aménagements (et donc pour les cartographier), mais elles révèlent l'état sanitaire des ouvrages, de même que les conflits entre les propriétaires. Au total, 38 pêcheries ont été identifiées dans les textes et sur les plans. Il convient cependant de rester prudent : les aménagements figurés sur ces plans existent bel et bien à la fin de l'époque moderne, mais ne peuvent pas être catégoriquement rattachés à cette seule période. Certains peuvent être plus anciens ou avoir subi des transformations au cours du temps sans qu'il soit possible d'en juger avec la documentation consultée.

Les plans dressés par G. Bouëssel illustrent l'aspect de ces pêcheries à l'aube du $\mathrm{XIX}^{\mathrm{e}} \mathrm{s}$. : certaines sont encore en état de fonctionnement, alors que d'autres sont en ruine. Elles sont également représentées avec un degré de précision qui permet d'en apprécier l'architecture, parfois imposante. Nombre d'entre elles sont construites en pierre et prennent la forme d'un barrage constitué de plusieurs piliers, séparés par des pertuis (fig. 8). À titre d'exemple, la pêcherie de Kerderrouarne à Bieuzy possède huit piliers encadrant sept pertuis, de même que la double pêcherie de Kermoret et de Goker à Pluméliau. La plus grande est celle de Tréblavet, entre Melrand et Saint-Barthélemy, avec douze pertuis, séparés au centre par le trou commun ${ }^{7}$ (fig. 9). Certaines d'entre elles sont fermées par un long

7. Les pêcheries qui barrent complètement le cours d'eau sont obligatoirement dotées d'un trou commun qui favorise le passage des poissons, à la remonte et à la descente, mais aussi des petites embarcations. Dans certains cas, ces établissements sont partagés entre plusieurs propriétaires et le trou commun constitue la limite de propriété. 


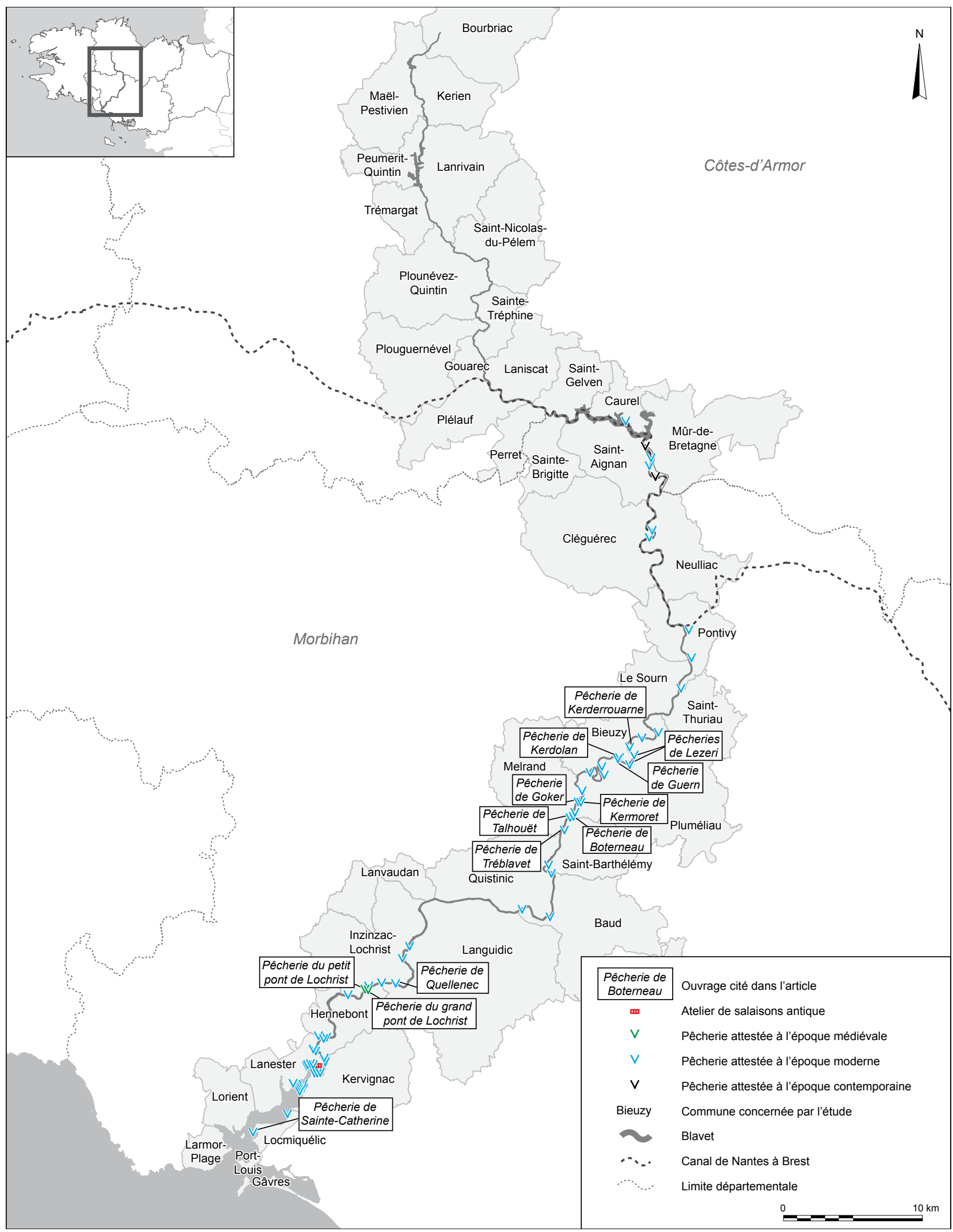

Figure 7 : Les pêcheries du Blavet (réal. P. Peter, source : arch. dép. Morbihan ; Langouët et al., 2008, p. 128).

Figure 7: The Blavet fisheries (С P. Peter, source: arch. dép. Morbihan; Langouët et al., 2008, p. 128). 
barrage linéaire, semblable à un môle ou à une digue, également en pierre et parfois doté d'ouvertures. La pêcherie de la Grand-Maison de Saint-Nicolas et celle de SaintGueltas à Bieuzy en sont l'illustration.

Ces ouvrages maçonnés sont en cela très différents des pêcheries médiévales mises au jour en 2005 sur la Loire, à Saint-Florent-le-Vieil. En effet, celles-ci possèdent une ossature en bois, vraisemblablement destinée à supporter des clayonnages, et leurs extrémités sont parfois renforcées à l'aide de blocs de pierre (Fillon, 2015, p. 121). D'autres, qui mêlent pierre et bois, prennent la forme de môle ou " duit », barrant presque entièrement le cours d'eau, et dans lequel sont aménagées des passes qui facilitent la capture des poissons. Si le principe fonctionnel de ces pêcheries fluviales reste le même - à savoir guider les animaux vers un piège positionné dans la partie la plus rétrécie de l'entonnoir - les différences en termes d'architectures et de modes de construction semblent plutôt liées au type de poissons pêchés et à l'anatomie générale du cours d'eau. D'autres rivières bretonnes, très fréquentées par ces espèces migratrices, présentent des aménagements similaires (Duhamel du Monceau, 1769-1782, vol. 2, section II, p. 254-255). Adaptées aux forts courants, les pêcheries de l'Ellé et de l'Isole près de Quimperlé sont ainsi, elles aussi, bâties en pierre (Martin, 2018, p. 314).

Si les plans du Blavet sont souvent très précis quant à la disposition spatiale de ces ouvrages, leur élévation n'est jamais décrite dans les archives modernes. Il faut donc s'intéresser aux autres cours d'eau armoricains, et notamment à l'Ellé, pour mieux comprendre l'architecture et le mode de fonctionnement de ces grandes pêcheries. Au début du XviII ${ }^{e}$ s., F. Le Masson du Parc, commissaire de la Marine et inspecteur général des pêches, évoque des aménagements composés de piliers "entièrement de maçonnerie ", dont l'intervalle est " clos de râteliers ». Il ajoute qu'il suffit de lever ces râteliers et d'y placer des nasses ou des filets pour capturer les saumons (Martin, 2018, p. 314). Les installations halieutiques du Blavet, très proches de cette description, semblent donc caractéristiques de ces pêcheries à saumon.

D'autres sont plus originales, comme les pêcheries de Kerdrolan et de Guern, entre Bieuzy et Pluméliau (fig. 8). L'ensemble est constitué de plusieurs murets, vraisemblablement en pierre, qui barrent les espaces compris entre les rives et deux petits îlots. Des ouvertures sont aménagées au sein de ces murets afin de poser les pièges. Enfin, d'autres pêcheries sont implantées entre les piles de certains ponts traversant la rivière ou à proximité des chaussées des moulins. C'est notamment le cas sur le site du moulin de Rimaison ou encore de Saint-Nicolas à Bieuzy.

Les propriétaires de ces établissements sont par ailleurs tenus de laisser un espace libre au sein de ces ouvrages afin de laisser remonter une partie des saumons : il s'agit $\mathrm{du}$ trou commun. Cette obligation d'assurer la circulation $\mathrm{du}$ poisson est mentionnée dès le $\mathrm{xv}^{\mathrm{e}} \mathrm{s}$. dans les archives de l'abbaye de la Joie à Hennebont et de nombreux litiges sont liés au bouchage de ce trou commun par les religieuses. C'est notamment le cas de la pêcherie qu'elles possèdent au-dessus de Lochrist et qui appartient pour moitié à la seigneurie de Guéméné (arch. dép. Morbihan, $60 \mathrm{H}$ 24). À titre d'exemple, les pêcheries de Kermoret et Goker et celle de Tréblavet sont, elles aussi, partagées entre deux propriétaires (fig. 8). L’espace ménagé par le trou commun était également mis à profit pour la navigation, quoi que limitée à de petites embarcations. Les témoignages et reconnaissances insistent sur les difficultés liées à la présence de ces nombreuses pêcheries car ces grands ouvrages, longs de plusieurs dizaines de mètres, entravaient la rivière.

La disposition des piliers pose la question de leur éventuel couronnement. À Orval, dans la Manche, un système de pont-pêcherie a été mis en évidence. Le pont est équipé, entre ses piliers, de râteliers (ou claies) de bois qui retiennent les poissons. Ces barrages amovibles sont vraisemblablement insérés dans des rainures prévues à cet effet (Canu et Billard, 2016, p. 596-597). Le pont en luimême ne semble pas servir au passage de véhicules mais plutôt à l'exploitation de la pêcherie. Sa forme est relativement proche des grandes pêcheries mises en évidence sur le Blavet, mais aucun document d'archives ne permet, pour l'heure, de le confirmer.

Pour finir, il est nécessaire de signaler que, contrairement à ce qu'annonçait $\mathrm{G}$. Bouëssel, toutes ces pêcheries n'ont pas été détruites dans leur intégralité. Dans son premier projet, il stipule que « les pêcheries de Boterneau [...] seront supprimées et rasées " (arch. dép. Morbihan, 3 S 373). Pourtant, d'après les recherches de J.-F. Nicolas, il subsiste encore aujourd'hui une partie de son barrage. D'autres vestiges sont ainsi encore conservés sous les eaux du Blavet : c'est le cas du radier de la pêcherie de Talhouët ou les ruines de celle de Quellenec (fig. 9). Ils témoignent du démantèlement partiel de ces établissements de pêche, dont il reste tout de même quelques traces, visibles lors de la mise à sec des biefs du canal (Nicolas et Cheyrouze, 2015, p. 51, 70).

\section{Les pêcheries de l'estuaire du Blavet}

Les pêcheries localisées dans l'estuaire sont soumises à un régime maritime souvent destructeur, impliquant de récurrentes réparations; elles sont toutefois difficilement datables par la seule lecture des archives. La plus ancienne pêcherie attestée dans cette zone appartenait au couvent Sainte-Catherine. Implantée dans l'anse de Locmiquélic, elle a été dessinée par F.-N. Baudot, dit 


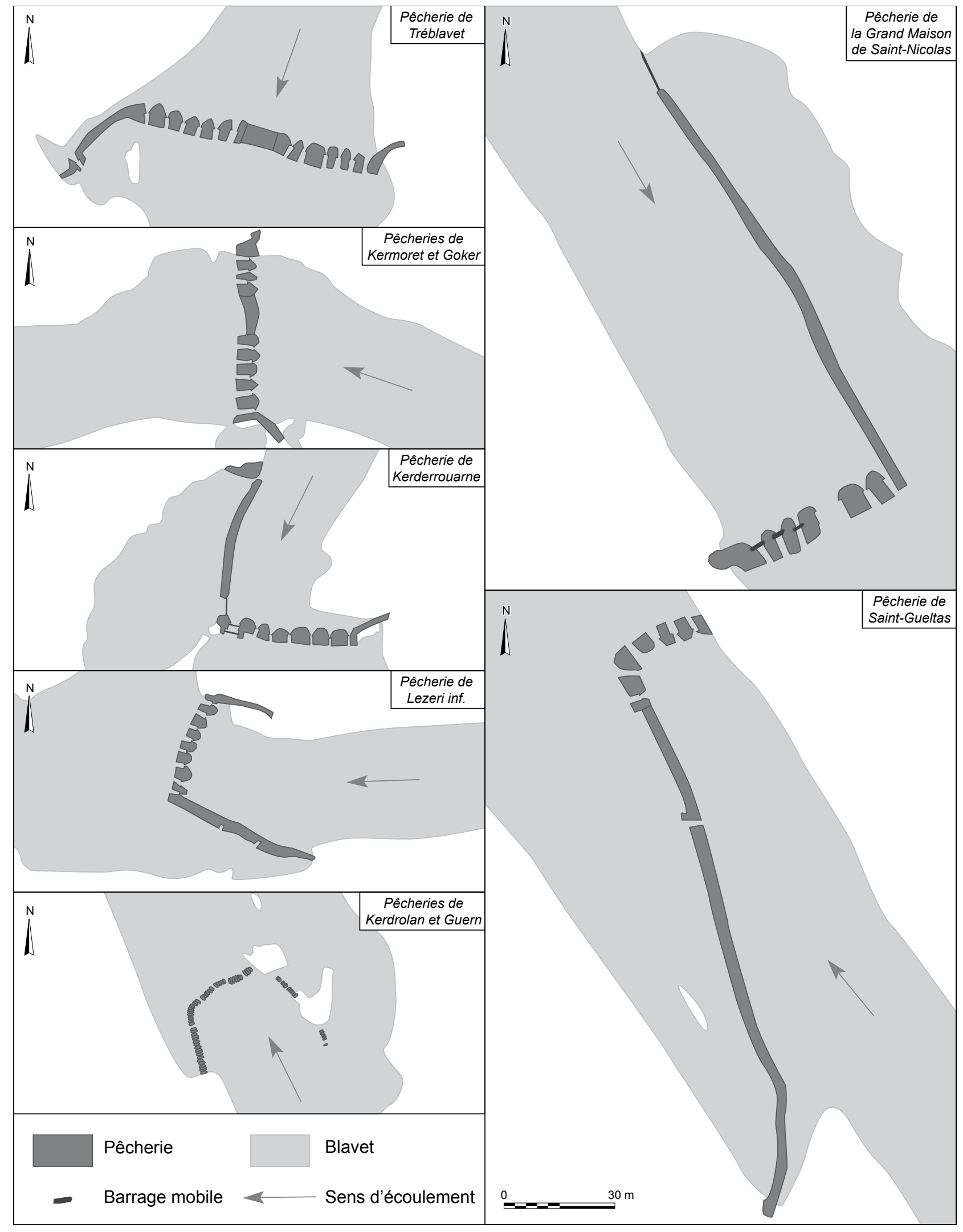

Figure 8: Quelques exemples de pêcheries identifiées sur le Blavet (réal. P. Peter, source : arch. dép. Morbihan, 35 374).

Figure 8: Examples of fisheries on the Blavet river (О P. Peter, source: arch. dép. Morbihan, 3S 374). 


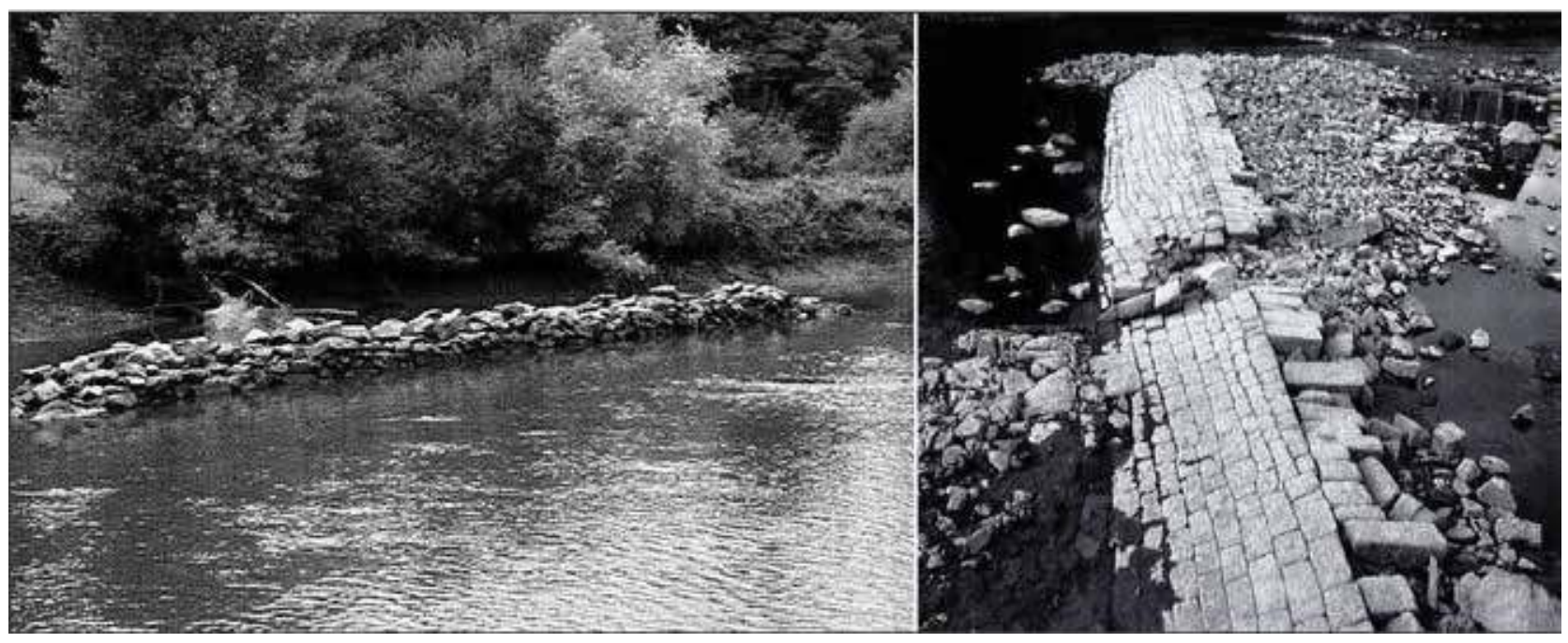

Figure 9: Vestiges de la pêcherie de Boterneau (à gauche) et de la pêcherie de Talhouët (à droite) en septembre 2013 (cliché J.F. Nicolas).

Figure 9: The Boterneau fishery (left) and the Talhouët fichery (right) in September 2013 (photo :J.-F. Nicolas).

Dubuisson-Aubenay, dans la première moitié du XVII ${ }^{\mathrm{e}} s$. (Croix, 2006, p. 293).

D'autres font l'objet d'une attention particulière dans les premières années du XviII ${ }^{\mathrm{e}} \mathrm{s}$. En effet, par lettres patentes datées du 23 avril 1726, le roi Louis XV mandate F. Le Masson du Parc pour se rendre sur place et inventorier les pièges à poissons présents dans l'estuaire (Phelypeaux, 1726, p. 2-3). Cet inventaire a un but : la suppression des ouvrages dont la prolifération a des conséquences non négligeables sur les richesses halieutiques des rivières bretonnes. Cette volonté de régulation du nombre de pêcheries s'inscrit dans une démarche amorcée par François Ir en 1544 et affirmée par un édit visant à réformer les eaux et les forêts du duché de Bretagne, depuis peu rattaché au royaume de France (Chasles, 1725, p. 151).

Le rapport remis en 1728 référence une trentaine de pêcheries, dont dix-sept sur le Blavet (fig. 10). Quelques précisions renseignent sur les matériaux employés : ce sont principalement les pêcheries en bois qui sont menacées (arch. dép. Morbihan, 9 B 257). Dix ans plus tard, le Conseil d'État du roi ordonne la destruction des pêcheries implantées illégalement sur le Scorff et le Blavet. Une nouvelle vérification porte leur nombre à vingtdeux et dix-huit d'entre elles seraient déjà abandonnées (arch. dép. Morbihan, 9 B 189). Toute pêcherie construite après 1544 (date de l'édit de François Ir) ou pour laquelle le propriétaire n'est pas en capacité de prouver l'ancienneté doit être démantelée. En 1757, l'abbaye de la Joie est menacée par ces suppressions et doit fournir les justificatifs concernant deux de ses pêcheries localisées à l'embouchure du Blavet depuis 1415 (Nicolas et Cheyrouze, 2015, p. 61).

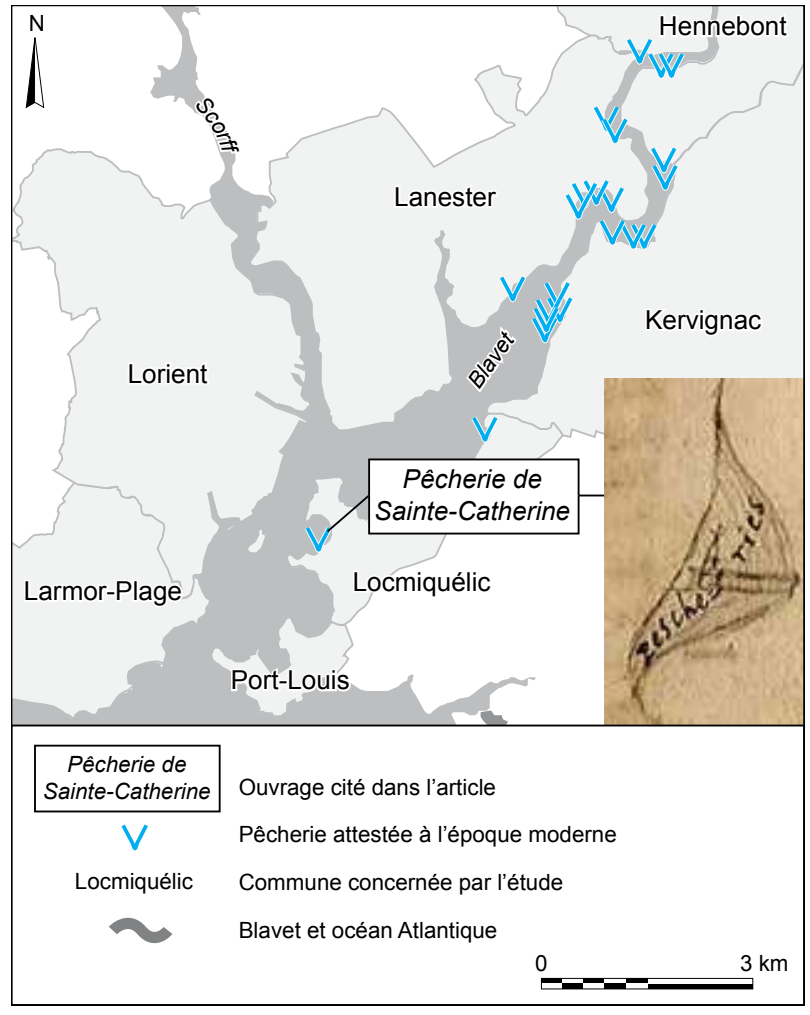

Figure 10 : Les pêcheries localisées dans l'estuaire du Blavet au XVIII' $S$. (réal. P. Peter, source : Langouët et al., 2008, p. 128).

Figure 10: Fisheries located on the Blavet estuary during the 18th century (C) P. Peter, source: Langouët et al., 2008, p. 128).

Dans son rapport, F. Le Masson du Parc décrit brièvement les pêcheries qu'il croise dans l'estuaire du Blavet. Il évoque des "bouchots", de même que des "parcs de clayonnage " (arch. dép. Morbihan, 9 B 189). Ces deux termes désignent des installations de pêche en bois : elles 
sont composées de deux alignements de pieux, régulièrement implantés et ensuite couverts d'un clayonnage (Langouët et al., 2008, p. 53). L'ensemble forme un V destiné à conduire les poissons dans la partie la plus rétrécie du dispositif. Ce dernier est agrémenté d'une nasse ou d'un filet terminal. L'anatomie de ces pièges à poissons est décrite avec grande précision par l'inspecteur général de la Marine Henri-Louis Duhamel du Monceau, dans le premier volume de son Traité général des Pesches (Duhamel du Monceau, 1769-1782, vol. 1, section II, p. 83-84). D'autres parcs, respectant le même principe architectural, sont construits en pierre. De tels aménagements sont connus sur le littoral normand et armoricain par exemple (Langouët et al., 2008; Billard et Bernard, 2016).

\section{Les espèces pêchées}

Les archives - et plus particulièrement celles de l'abbaye de la Joie à Hennebont - offrent un aperçu des espèces piégées par ces pêcheries. Il s'agit essentiellement d'espèces migratrices qui, pour certaines, remontent le cours d'eau pour se reproduire comme le saumon, alors que d'autres, comme les anguilles, descendent le fleuve en direction de la mer. Ainsi, en 1479, le duc de Bretagne signale que des saumons, des lamproies ou encore des aloses, sont illégalement pêchés (arch. dép. Morbihan, $60 \mathrm{H}$ 21). Les anguilles sont particulièrement appréciées et collectées lors de leur migration vers l'estuaire, entre octobre et novembre (Nicolas et Cheyrouze, 2015, p. 53). La multiplication des barrages pose bien évidemment des problèmes pour le maintien des populations, de saumons notamment. Cet appauvrissement motive en partie la décision, dans le courant du $\mathrm{XVIII}^{\mathrm{e}}$ s., de supprimer les établissements de pêche jugés illégaux sur les cours d'eau et le littoral breton. Il est également à l'origine de plaintes, consignées dans les archives.

S’il n'est pas possible de déterminer les quantités de poisson pêché, quelques textes apportent toutefois des éléments de réponse, principalement en ce qui concerne le saumon. En 1860, l'ingénieur J.-P.-A. Le Helloco précise qu'avant la canalisation du Blavet, le saumon pouvait remonter jusqu’à $90 \mathrm{~km}$ de son embouchure et que les pêcheurs rapportaient quotidiennement jusqu'à une trentaine de poissons (arch. dép. Morbihan, 3 S 633). Cette abondance était donc à la fois profitable aux propriétaires de pêcheries mais aussi aux pêcheurs au filet donc l'activité échappe à notre étude.

\section{Les moulins hydrauliques}

Ces dernières décennies, les fouilles archéologiques de moulins antiques ou médiévaux ont considérablement enrichi les connaissances à ce sujet (Jaccottey et Rollier,
2016). Contrairement aux pêcheries, dont aucune n'a été identifiée sur le haut Blavet, les moulins sont représentés sur la totalité du fleuve, estuaire excepté. Au total, 32 ouvrages ont été recensés (13 sur le canal actuel, 9 sur la section commune avec le canal de Nantes à Brest et 10 sur le haut Blavet) ainsi qu'un dernier, non localisé (fig. 11). Ils sont répartis de façon assez hétérogène à l'échelle du territoire, mais sont globalement situés à l'entrée ou à la sortie d'un méandre, la configuration du cours d'eau influant sur la puissance du courant.

\section{Les moulins médiévaux}

La plus ancienne mention d'une activité meunière sur le Blavet est datée - comme pour la pêche - de 1125, le seigneur de Castennec mettant son moulin à la disposition des paroissiens de Bieuzy. Ce moulin, aujourd'hui disparu, était probablement localisé près de la chapelle Saint-Gildas.

Tout au long du Moyen Âge, la puissante famille de Rohan dispose ou octroie de nombreux moulins sur le Blavet. Dès la fin du XII ${ }^{e}$ s., le vicomte Alain III de Rohan fait ainsi don de terres à l'abbaye de Bon Repos, afin d'y installer pêcheries et moulins (Nicolas et Cheyrouze, 2015, p. 23). Le moulin de Guernal (ou du Hâle), près de Pontivy, existe avant 1324, comme l'indique un accord passé entre le vicomte de Rohan et le seigneur de Quénécan. D’autres moulins sont également attestés à Pontivy à la même période. Ces vieux moulins, construits à la demande de l'influente famille, sont alimentés par un bief de dérivation encore conservé et prolongé lors de la canalisation du cours d'eau. Ils sont réputés pour abriter les plus importantes meules à blé du duché et figurent sur la carte de Cassini (Éveillard, 1984, p. 19). Un aveu énumérant les possessions des Rohan, daté de 1471, fait état de plusieurs établissements, dont le moulin de Saint-Nicolas à Bieuzy ou encore le moulin du Roh à Saint-Thuriau, qui figure lui aussi sur la carte de Cassini (Éveillard, 1984, p. 13). C'est aussi le cas du moulin de Rimaison, dont la construction est autorisée par le vicomte de Rohan en 1512. L'inscription sur le pignon ouest date l'édifice de 1556, ou plutôt sa rénovation. En effet, la seigneurie de Rimaison dispose de moulins sur le Blavet au moins depuis le XIv ${ }^{e}$ s. (Nicolas et Cheyrouze, 2015, p. 28).

L'abbaye de la Joie possède également des moulins, installés sur un bief de dérivation. Leur création est octroyée par Jean I ${ }^{\text {er }}$ au XIII ${ }^{e}$ s. (Nicolas et Cheyrouze, 2015, p. 39). C'est par exemple le cas des moulins du Grand Barrage et des Gorets. Il est également question d'un « moulin d'en bas du bois du duc ", qu'il n'a pas été possible de localiser avec précision. 


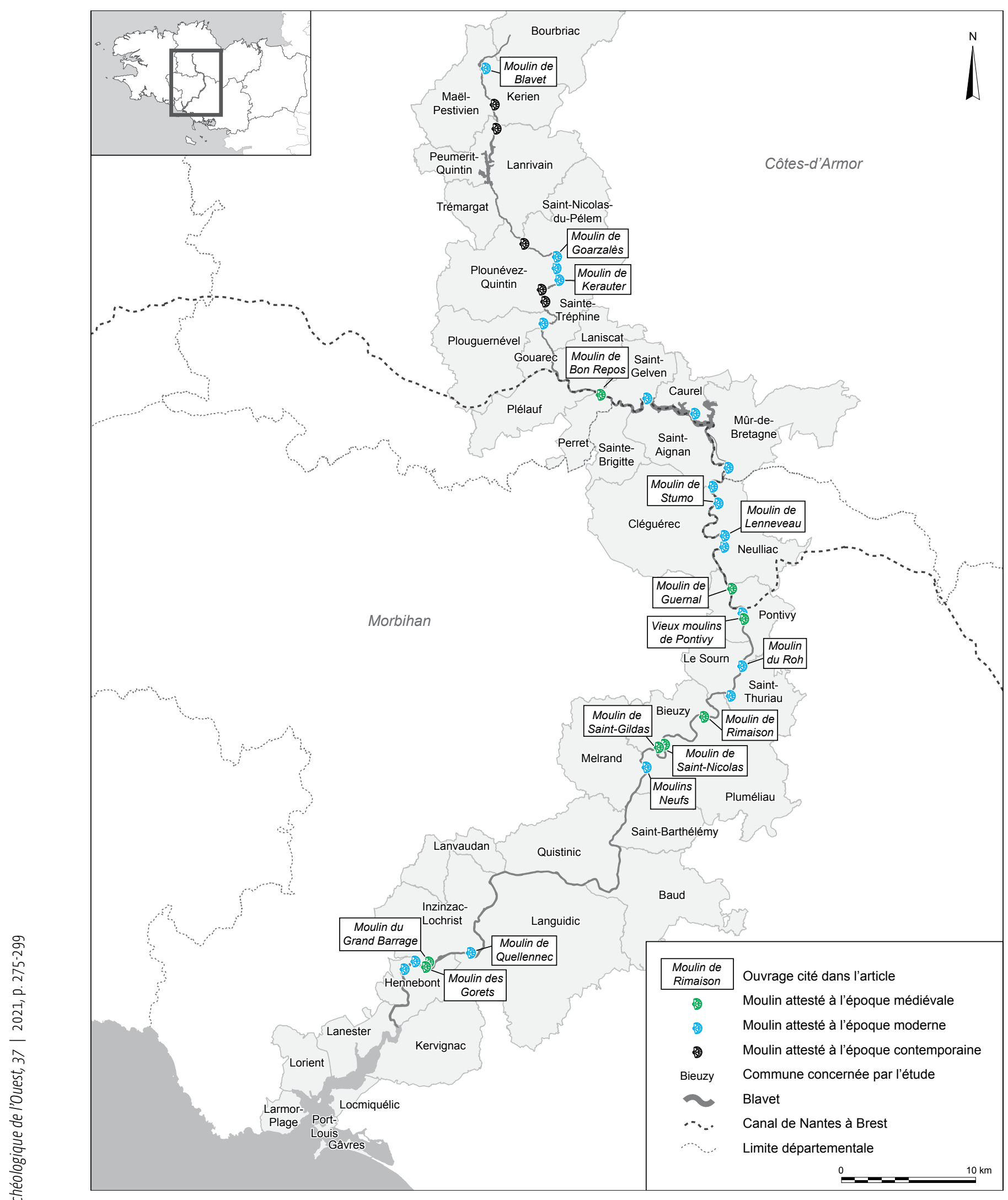

Figure 11 : Les moulins du Blavet (réal. P. Peter, source : arch. dép. Morbihan).

Figure 11: Watermills on the Blavet (৫ P. Peter, source: arch. dép. Morbihan). 
Au total, 8 moulins médiévaux ont pu être localisés sur le Blavet. À défaut d'apporter plus de renseignements, la documentation révèle toutefois l'état de ces installations, certaines étant laissées à l'abandon. En 1474, le duc François II s'inquiète de la dégradation des moulins " du duc " et les cède à l'abbaye Notre-Dame de la Joie. Un siècle plus tard, ils semblent toujours en ruine et il "n'en reste que le bardeau, conduit et chaussée " (arch. dép. Morbihan, $60 \mathrm{H}$ 19). Les religieuses sont autorisées à reconstruire ces moulins et même à les déplacer (arch. dép. Morbihan, 60 H 27).

\section{Les moulins attestés aux époques moderne et contemporaine}

Aux ouvrages médiévaux s'ajoutent 19 moulins connus à l'époque moderne et 5 à l'époque contemporaine. Le site des Moulins Neufs de Pluméliau, dont le nom laisse supposer qu'ils sont héritiers d'un établissement plus ancien, est attesté au moins depuis le XviII ${ }^{\mathrm{e}}$ s. et détruit en 1847 (arch. dép. Morbihan, 3 S 448). Les vestiges du déversoir et de l'arrivée d'eau, bâtis au moyen de gros blocs de pierre, sont actuellement toujours visibles, lorsque le bief dans lequel ils se trouvent est mis à sec (fig. 12). D'autres chaussées de moulins sont encore conservées. C'est par exemple le cas de la chaussée de Quellenec. Leur préservation témoigne des choix faits par les ingénieurs pour canaliser le cours d'eau, puisque seuls les aménagements situés dans le chenal de navigation sont menacés. Le haut Blavet est lui aussi doté de plusieurs moulins, pour certains mentionnés sur la carte de Cassini, notamment ceux de Kerauter, de Goarzalès ainsi que celui du Blavet - le plus septentrional - qui profite des eaux de l'étang du même nom.
La lecture de la carte d'état-major et du cadastre napoléonien a permis d'identifier d'autres moulins, vraisemblablement mis en place dans la première moitié du $\mathrm{XIX}^{\mathrm{e}} \mathrm{s}$. Sans grande surprise, aucune création n'est à signaler sur l'actuel canal du Blavet et sur la section commune avec le canal de Nantes à Brest, la tendance étant plus à la destruction qu'à la construction en raison de la canalisation du cours d'eau à la même période.

\section{Les ressources produites aux époques médiévale et moderne}

À l'époque moderne, les moulins du Blavet sont tous dotés de roues à aubes et ils disposent aussi d'un ou plusieurs corps de bâtiments, renfermant un ou plusieurs couples de meules (Nicolas et Cheyrouze, 2015, p. 23-25) [fig. 12]. Ces moulins semblent d'ailleurs actifs toute l'année, comme l'atteste $\mathrm{G}$. Bouëssel en 1802, expliquant que " les deux tournants des moulins à froment de Pontivy tournent sans cesse nuit et jour, même dans les plus grandes sécheresses " (arch. dép. Morbihan, 3 S 373). Il en va de même pour le moulin de Saint-Nicolas à Bieuzy, pour lequel il suffisait de condamner momentanément les pêcheries afin de disposer de suffisamment d'eau pour alimenter les roues (arch. dép. Morbihan, S1245). Les archives apportent également quelques informations sur les céréales utilisées : froment, blé noir, seigle, mil, etc. Plusieurs espèces peuvent d'ailleurs être transformées à l'échelle d'un même établissement. Par exemple, les moulins de Rimaison à Bieuzy sont composés de trois bâtiments : le moulin à blé noir et à seigle, reconstruit au $\mathrm{XVI}^{\mathrm{e}} \mathrm{s}$., le moulin à froment et le moulin à mil (Nicolas et Cheyrouze, 2015, p. 28-30). Ces installations ne servaient
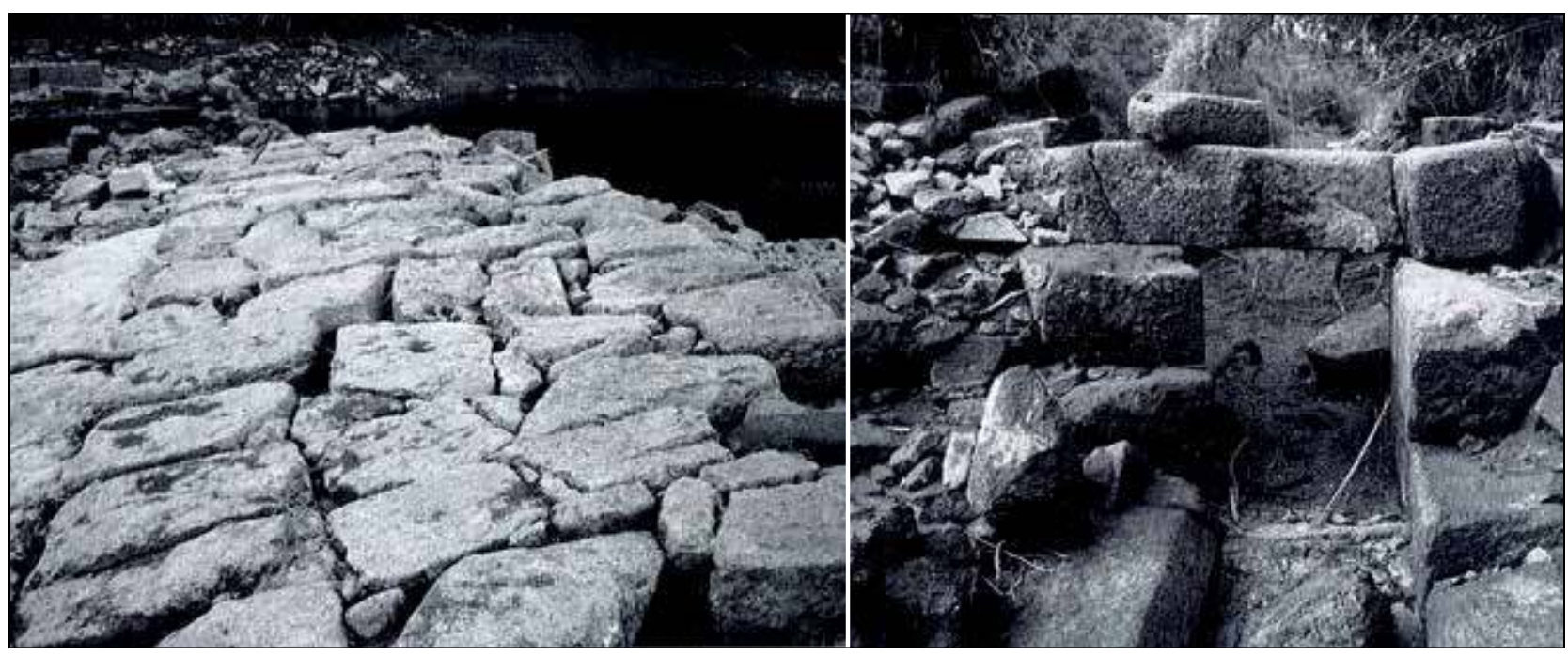

Figure 12 : Vestiges de la chaussée et de l'arrivée d'eau des Moulins-Neufs en septembre 2013 (cliché J.-F. Nicolas).

Figure 12: Remains of the roadway and the Moulins-Neufs water inlet in September 2013 (photo J.-F. Nicolas). 
pas uniquement à la production de farine : par exemple, les Moulins Neufs de Pontivy étaient aussi utilisés pour produire du tan, poudre d'écorce de chêne, employé pour le tannage des peaux (arch. dép. Morbihan, 3 S 373).

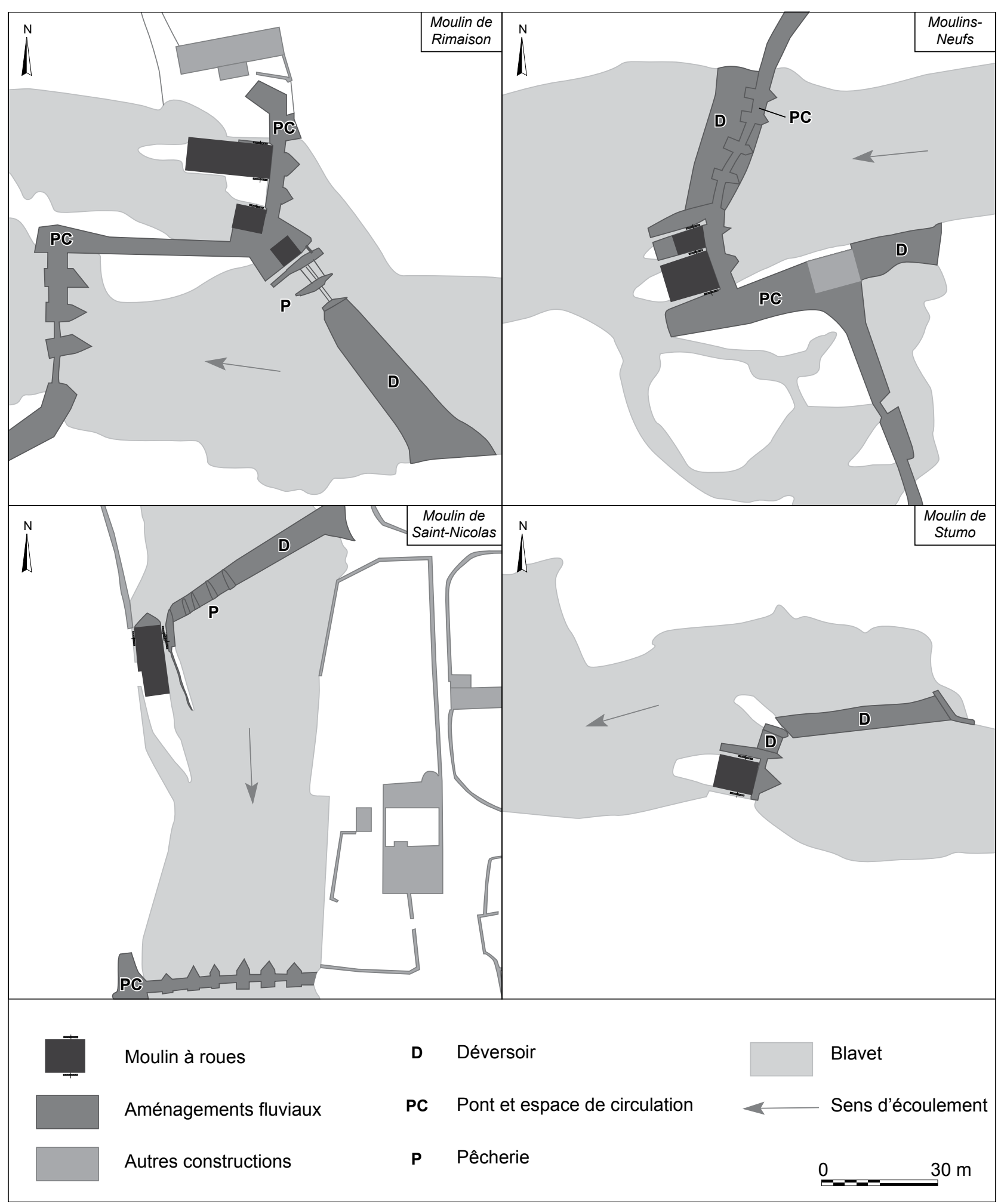

Figure 13 : Quelques exemples de moulins identifiés sur le Blavet (réal. Peter, source : arch. dép. Morbihan, 35 374; Arch. nat. : F14 10088.1.3). Figure 13: Some of the mills on the Blavet river (๑ P. Peter, source: arch. dép. Morbihan, 3S 374; Arch. nat.: F14 10088.1.3). 


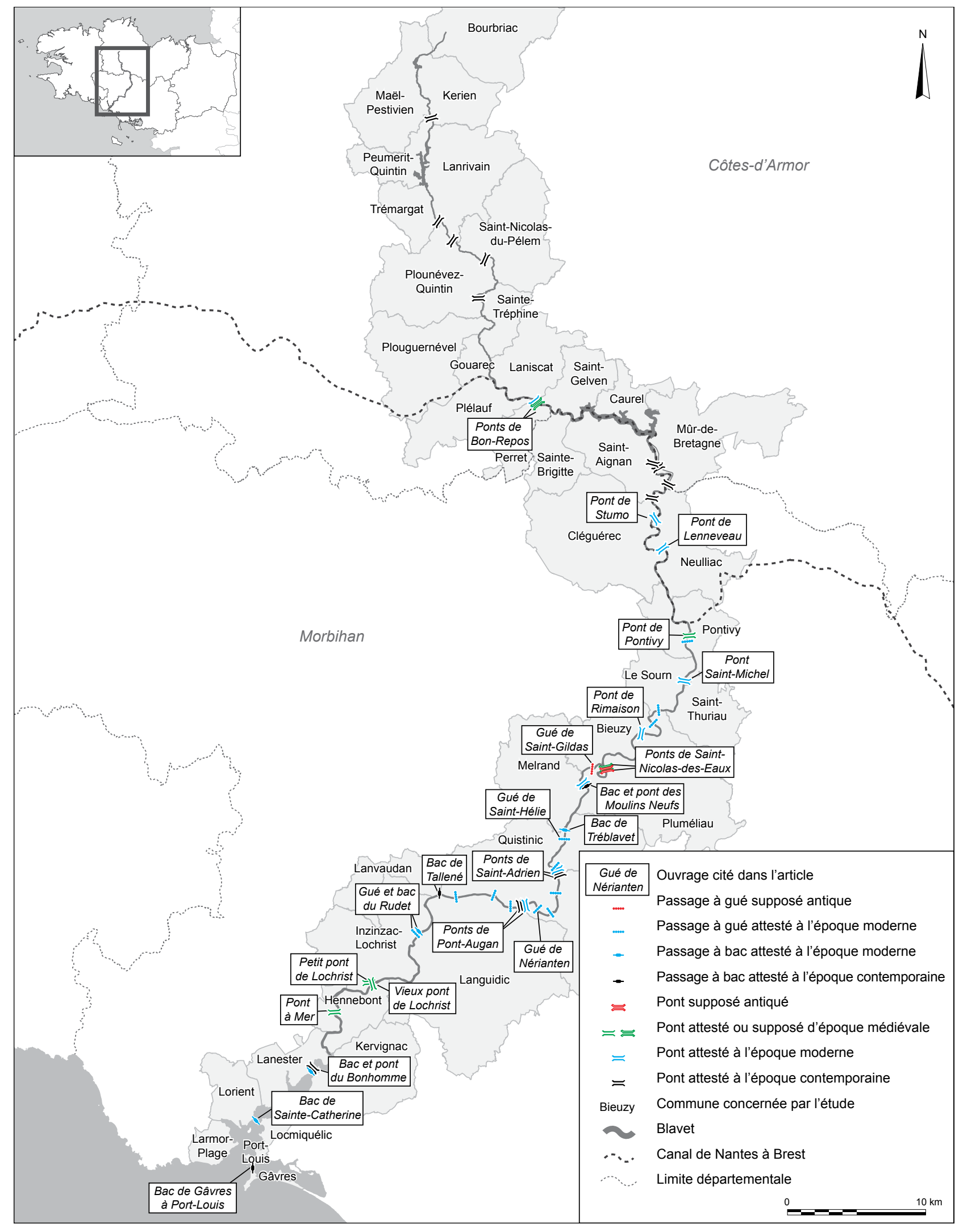

Figure 14 : Les sites traversiers du Blavet : passages à gué, à bac et ponts (réal. P. Peter, source : arch. dép. Morbihan).

Figure 14: Ford crossing, boat crossing and bridges on the Blavet (० P. Peter, source: arch. dép. Morbihan). 


\section{Les sites traversiers}

\section{Les passages à gué}

De tous les types de franchissement, le passage à gué est celui qui demande le moins d'investissement et qui est le plus répandu à l'échelle de nombreuses rivières, pour des époques où le pont ne constitue pas le principal mode de communication entre les rives (fig. 14). Ce " hautfond naturel utilisé par l'homme pour traverser un cours d'eau " est utile non seulement pour assurer la circulation des hommes, mais aussi celle du bétail et des véhicules tractés par des animaux (Dumont, 2002, p. 29). À l'instar du pont, le passage à gué revêt un rôle socio-économique important. Si la configuration de ces hauts-fonds peut offrir de nombreux points de passage, ceux-ci sont toutefois conditionnés par les aléas météorologiques et les saisons, la traversée étant impossible en période de crue. Sur le Blavet, plusieurs passages à gué ont été relevés. Dans les rapports des ingénieurs, certains qualifient de passages ce que d'autres appellent hauts-fonds. L'analyse du paysage et de la toponymie peut, dans une moindre mesure, apporter, là, des éléments de réponses. L'ingénieur $\mathrm{G}$. Bouëssel ne cite pas toujours le même nombre de passages à gué - sept ou neuf selon les documents - alors qu'au moins dix structures sont repérables sur les plans dressés par son équipe, en 1803. Quelques années plus tard, l'ingénieur M. Poterel en mentionne également neuf, mais qui ne correspondent pas tous à ceux évoqués par $\mathrm{G}$. Bouëssel.

Pour l'époque romaine, il n'existe aucune véritable attestation de passage à gué sur la rivière. Cependant, la présence de l'agglomération secondaire de Castennec à Bieuzy (connue sous le nom de Sulim sur la Table de Peutinger), invite à envisager l'existence de points de passage à cette période (fig. 15). En effet, la traversée du cours d'eau par deux voies romaines - les voies de Vannes à Carhaix et de Rennes à Quimper - peut aller dans ce sens, même si les deux axes n'empruntaient peut-être qu'un seul passage (Galliou, 2009, p. 83). Non loin de là se trouve le site de Saint-Gildas (ou SaintGueltas) que G. Bouëssel identifie comme haut-fond, alors que $\mathrm{M}$. Poterel le désigne comme passage à gué à part entière (arch. dép. Morbihan, S 124). Si rien ne permet d'être affirmatif, la proximité du lieu avec l'éperon de Castennec est cependant intéressante : en effet, il donne accès à la partie rétrécie du promontoire. Le passage d'autres voies romaines, au nord de la zone d'étude, suggère la présence d'éventuels franchissements, mais il n'est pas possible d'en dire plus en l'état actuel des recherches.

À l'époque moderne, douze à seize hauts-fonds peuvent avoir fait office de passages à gué. La plupart assure la connexion entre deux axes de communication, comme les gués de Saint-Hélie ou encore de Nérianten (fig. 16). Plusieurs d'entre eux sont d'ailleurs remployés pour la construction des déversoirs des écluses, en raison de leur configuration naturelle mais aussi de leur importance au sein de la rivière. Les annotations ajoutées aux plans de 1803 montrent que nombre de ces passages sont destinés à être détruits par le creusement du canal, ce qui explique en partie leur absence du cadastre napoléonien, relevé quelques années plus tard.

Si ces passages à gué sont attestés à l'époque moderne, une origine médiévale peut être envisagée pour plusieurs d'entre eux. C'est le cas du gué du Rudet : ce point de franchissement, doublé d'un bac, est principalement utilisé par les habitants pour se rendre aux foires et marchés en période de basses eaux (Nicolas et Cheyrouze, 2015,

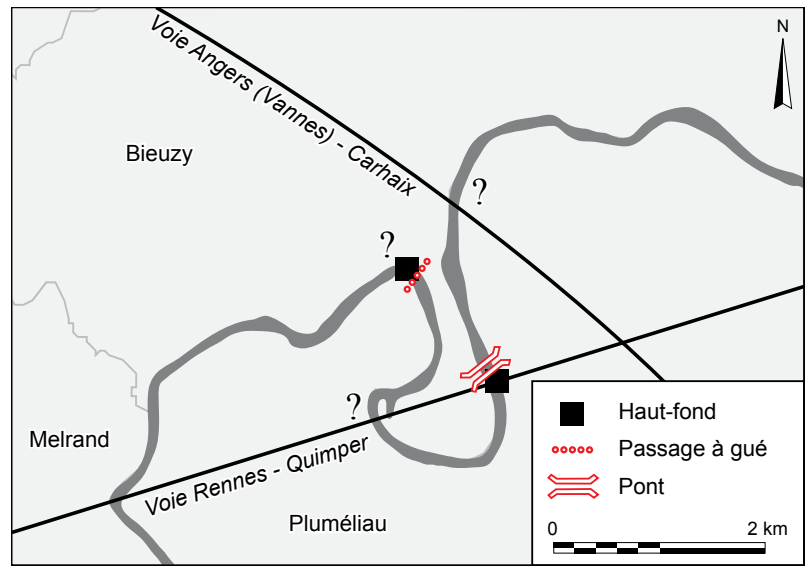

Figure 15 : Hypothèses de franchissement dans le secteur de Castennec à Bieuzy (réal. P. Peter, source : arch. dép. Morbihan, voies romaines d'après T. Lorho et M. Monteil).

Figure 15: Possible crossing points in the Castennec to Bieuzy sector (C) $P$. Peter, source: arch. dép. Morbihan, Roman roads after T. Lorho and $M$. Monteil).

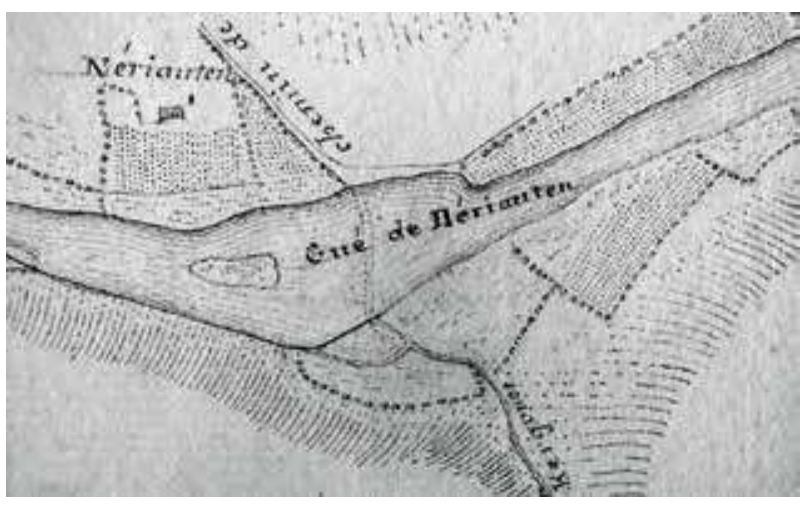

Figure 16 : Le passage à gué de Nérianten sur un plan de 1803 (cliché P. Peter, source : arch. dép. Morbihan, 3 S 374).

Figure 16: Ford crossing at Nérianten on the 1803 map (photo P. Peter, source: arch. dép. Morbihan, 35374). 
p. 46). Aucune mention de passages à gué n'a en revanche été découverte pour la section commune avec le canal de Nantes à Brest et pour le haut Blavet.

\section{Les passages à bac}

L'usage d'un bac pour franchir le cours d'eau est attesté sur le Blavet. En revanche, leur nombre est assez faible, à l'image de ce qui a été observé sur le Cher (fig. 14). La faible représentativité de ceux-ci ne doit cependant pas tromper : ne pas être figuré ne signifie pas obligatoirement ne pas avoir existé (Mauret-Cribellier et Schemmama, 2013, p. 158). Sur l'actuel canal du Blavet, deux des trois passages à bac référencés - soit le Rudet et Tallené - ne sont pas figurés sur les plans de 1803. En revanche, ils sont cités par A. Laumailler en 1785 et par G. Bouëssel en 1802. Ils figurent également sur le cadastre napoléonien sous le nom de " passage ». Il s'agit là d'exemples de passages à gué doublés d'un bac, permettant de traverser la rivière lorsqu'elle n'est pas guéable. Ces aménagements sont susceptibles d'être plus anciens, mais rien ne permet de le prouver (le bac de Tallené apparait dans les archives au moins depuis 1643). Pour la partie estuarienne du Blavet, deux autres passages à bac ont été inventoriés : le bac de Sainte-Catherine, qui assure la liaison entre Locmiquélic et Lorient, et celui du Bonhomme entre Kervignac et Caudan, avant la construction du pont du Bonhomme en 1974. Ces deux ouvrages sont cités dans un document daté du 5 août 1804 (arch. dép. Morbihan, $3 S$ 256).

Le bac de Tréblavet est, quant à lui, attesté à l'époque contemporaine. Le montant de la traversée, fixé le 26 avril 1852, suggère une mise en service à cette période. Un autre passage à bac est suggéré par J.-D. Éveillard et serait créé en réponse à la destruction du pont des Moulins Neufs, en 1808 (Éveillard, 1984, p. 28). Enfin, une liaison plus récente existe dans l'estuaire, entre Gâvres et Port-Louis.

\section{Les ponts}

Jusqu'au XIX ${ }^{\mathrm{e}}$ s., les ponts sont encore peu nombreux et sont majoritairement édifiés en bois et en pierre, malgré l'introduction progressive du métal dans ce type de construction (Wouters et Wibaut, 2019, p. 581). À l'instar des moulins, les découvertes de ponts sont de plus en plus nombreuses et apportent un nouvel éclairage sur l'anatomie de ces constructions (Barruol et al., 2011; Dumont et al., 2014, par exemple). À la veille de sa canalisation, 21 ponts ont été recensés sur le Blavet (9 sur l'actuel canal, 7 sur la section commune avec le canal de Nantes à Brest et 5 sur le haut Blavet), auxquels s'ajoutent plusieurs ouvrages supposés et/ou non localisés (fig. 14).
Plusieurs hypothèses ont été formulées concernant l'établissement de ponts dès l'époque romaine. La première concerne un pont en bois, qui aurait été édifié à Saint-Nicolas-des-Eaux afin d'assurer le franchissement de la rivière par la voie de Rennes à Quimper. Il aurait ainsi permis d'accéder à l'éperon de Castennec, en complément des passages à gué évoqués plus haut (Galliou, 2009, p. 83). La seconde, encore moins évidente, est celle d'un autre pont en bois dans le secteur de l'abbaye de Bon Repos à Saint-Gelven (Naas, 1999, p. 159). Il serait situé sur le tracé des voies menant de Lamballe à Priziac. Si leur emplacement supposé est plausible en raison de la présence d'un important réseau viaire à l'époque romaine, leur existence n'a pour l'heure jamais été attestée.

Le pont le plus ancien, daté de l'époque médiévale, pourrait être localisé à Pontivy. Selon une tradition orale très incertaine, il aurait été bâti à l'initiative du moine Ivy au VII ${ }^{\mathrm{e}}$ s., après la construction de son monastère sur la rive gauche du Blavet, vers 686 (Le Mené, 1982, p. 214). En bois, il aurait ensuite été remplacé par un pont en pierre, décrit par F.-N. Dubuisson-Aubenay en 1636, qui évoque un pont de pierre à cinq arches (Croix, 2006, p. 272). Les ponts d'Hennebont et de Lochrist ont également une origine médiévale. Le premier, probablement plus ancien, apparaît dans les textes à partir du XIII ${ }^{\mathrm{e}}$ s. sous l'appellation " pont à mer" (Nicolas et Cheyrouze, 2015, p. 45). Il est aussi mentionné par F.-N. Dubuisson-Aubenay qui signale un pont à six arches, fait de pierre et couvert de pièces et planches de bois (Croix, 2006, p. 278). Les ponts de Lochrist sont également attestés depuis le XIII ${ }^{\mathrm{e}}$ s. dans les archives de l'abbaye de la Joie, puisqu'ils supportent plusieurs pêcheries. Comme le suggèrent son nom et son état de dégradation précoce, le Vieux Pont - qui est aussi le plus court avec cinq arches - pourrait être antérieur au second. Enfin, le pont de Rimaison, associé au moulin reconstruit au milieu du XVI ${ }^{\mathrm{e}}$ s., est doté de quatre arches et relie la rive gauche du Blavet aux moulins de Rimaison, qu'il faut traverser pour atteindre la rive droite. Enfin, l'actuel pont de l'abbaye de Bon Repos à Saint-Gelven, daté du XVII ${ }^{e}$ s., remplace très probablement un franchissement médiéval (Benferhat et al., 1999, p. 95).

À ces constructions s'ajoute le pont de Saint-Nicolasdes-Eaux, bâti en pierre et en bois, et signalé en ruine avant 1753. Il serait utilisé depuis longtemps pour se rendre aux foires et marchés de la région (arch. dép. Morbihan, S 2518). Dans les faits, rien n'indique qu'il puisse dater de l'époque médiévale, mais son emplacement invite à le supposer.

À l'instar des pêcheries, les plans d'ingénieur offrent une vision assez précise des ponts, du point de vue de leur architecture et de leur position au sein de la rivière à la fin de l'époque moderne. Entre Pontivy et Hennebont, 
quatre ouvrages ont été recensés : les ponts des Moulins Neufs, de Saint-Michel, de Saint-Adrien et du PontAugan. Le premier, doté de huit arches et d'un tablier en bois, s'écroule en 1808. Le pont de Saint-Michel dispose de dix arches, dont l'une accueille une pêcherie. Le troisième, localisé en amont du pont actuel, est composé de huit arches en pierre, dont quatre étaient écroulées à la fin du XviII ${ }^{e}$ s. Enfin, le Pont-Augan présente sept arches et rejoint un second franchissement destiné à traverser l'Ével (arch. dép. Morbihan, S 2518). La crue dévastatrice de 1642 peut être tenue pour responsable d'une partie des destructions subies par les ponts du Blavet (Nicolas et Cheyrouze, 2015, p. 77). En outre, plusieurs textes évoquent l'usage comme carrière des ponts à l'état de ruine, qui sont activement démantelés pour leurs matériaux lors de la canalisation de la rivière. La section commune avec le canal de Nantes à Brest et le haut Blavet sont moins bien renseignés. Les ponts de Lennevau et de Stumo, tous deux liés à un moulin, sont attestés à l'époque moderne.

La plupart des ponts contemporains ont été érigés lors de la création du canal, en réponse au développement de plus en plus intense du réseau routier. L'édification du pont du Bonhomme en 1974, remplaçant par ailleurs le passage à bac du même nom, en est un exemple. Un texte daté de 1878 fait également état de quatre autres ponts entre celui de Stumo et l'actuel barrage de Guerlédan (arch. dép. Morbihan, 3 S 262). Le haut Blavet possède quant à lui cinq ponts figurant sur le cadastre napoléonien et d'autres documents datés du XIX ${ }^{e}$ s.

\section{Les écluses}

Symbole par excellence de la maîtrise des eaux fluviales, l'écluse à sas est une construction bien particulière. Contrairement aux ouvrages précédemment cités, elle est intrinsèquement liée à la canalisation de la rivière et donc contemporaine de celle-ci. Elle représente l'aboutissement d'une longue expérimentation, dont l'unique but est d'asservir la rivière et de l'adapter aux besoins de l'homme. Sa diffusion en Europe à partir du $\mathrm{Xv}^{\mathrm{e}} \mathrm{s}$. annonce le début d'une nouvelle ère fluviale (Violet, 2004, p. 287-289). Grâce à elle, il est désormais possible de franchir les lignes de crête pour faire communiquer deux bassins versants et ainsi assurer une connexion pérenne jusqu'alors impossible, malgré de nombreux tâtonnements et tentatives. Encore peu étudiées par l'archéologie, ces écluses le sont surtout par les historiens et historiens des techniques, qui s'intéressent aux grands canaux de navigation.

Sur le Blavet, toutes les écluses sont construites entre 1804 et 1840 (fig. 17). Au nombre de 28 entre Hennebont et Pontivy, elles sont complétées par 32 autres ouvrages qui assurent la connexion avec le canal de Nantes à Brest. Parmi elles, 12 écluses appartiennent à la partie désaffectée du canal, condamnées par la construction de l'usine hydroélectrique de Guerlédan et de son lac, qui a englouti les 18 suivantes. Les écluses de Bon Repos et de Saint-Hervé marquent l'arrêt du parcours sur le Blavet, avant que le canal n'emprunte le ruisseau du Doré pour atteindre le point de partage de Glomel et poursuivre sa course jusqu’à Brest.

Le choix des emplacements de ces écluses n'a rien d'hasardeux. En effet, les reconnaissances réalisées avant la canalisation ont permis d'identifier les secteurs les plus propices à leur implantation, tout en profitant des aménagements préexistants pour asseoir les ouvrages. Certaines écluses sont construites à même la berge, alors que d'autres nécessitent la mise en place d'une dérivation, qui a eu pour principale conséquence l'élargissement du cours d'eau et la formation d'îles. Certains hauts-fonds et même quelques passages à gué, comme celui du Rudet (entre Inzinzac-Lochrist et Languidic), peuvent servir d'assise. Ce sont surtout les chaussées de moulins qui sont remployées et adaptées à la nouvelle construction. Cette superposition entraîne une complexification de ces sites, qui peuvent à la fois réunir moulin, pêcherie et écluse, comme c'est le cas du moulin de Rimaison (fig. 13).

\section{LA CANALISATION ET SES CONSÉQUENCES SUR LA CONSERVATION DU SIGNAL ARCHÉOLOGIQUE}

D’un point de vue général, la création du canal du Blavet et le relèvement de son plan d'eau ont eu une incidence majeure sur l'intégrité des aménagements fluviaux. Les reconnaissances dans le but de canaliser le cours d'eau sont formelles : les ouvrages cités sont destinés à être détruits. Sur les plans, les espaces à curer - dragages qui éventrent hauts-fonds guéables, chaussées et pêcheries sont figurés, et les entités à supprimer sont indiquées. Les recherches en archives et les compléments apportés par J.-F. Nicolas révèlent que la canalisation a été réalisée dans le respect des plans établis, mais en ne portant atteinte aux aménagements que lorsque ceux-ci sont localisés dans le chenal de navigation. Les vestiges observés lors de l'assec des biefs du Blavet, parfois bien conservés, invitent à reconsidérer le rôle destructeur de la canalisation sur le patrimoine fluvial. Ainsi, les pêcheries ne sont pas intégralement détruites puisqu'il subsiste des traces de ces aménagements dans le cours d'eau. Il en va de même pour les chaussées de certains moulins.

Enfin, au regard de l'implantation de certaines écluses, il est possible que des maçonneries ou des vestiges de franchissement soient encore partiellement préservés, nombre d'entre elles ayant été installées au contact d'aménage- 


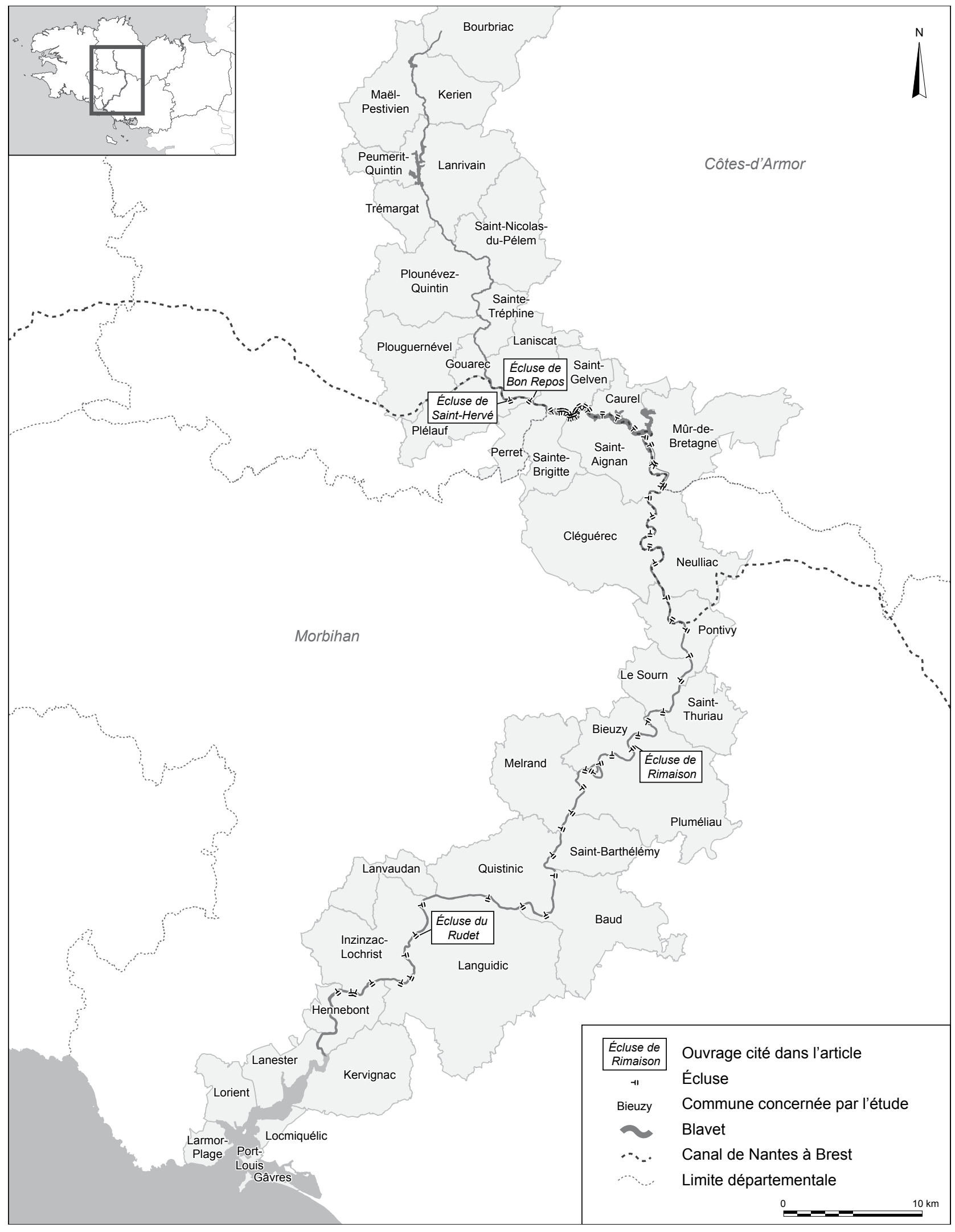

Figure 17 : Les écluses du Blavet (réal. P. Peter, source : arch. dép. Morbihan).

Figure 17: The locks on the Blavet (৫ P. Peter, source: arch. dép. Morbihan). 


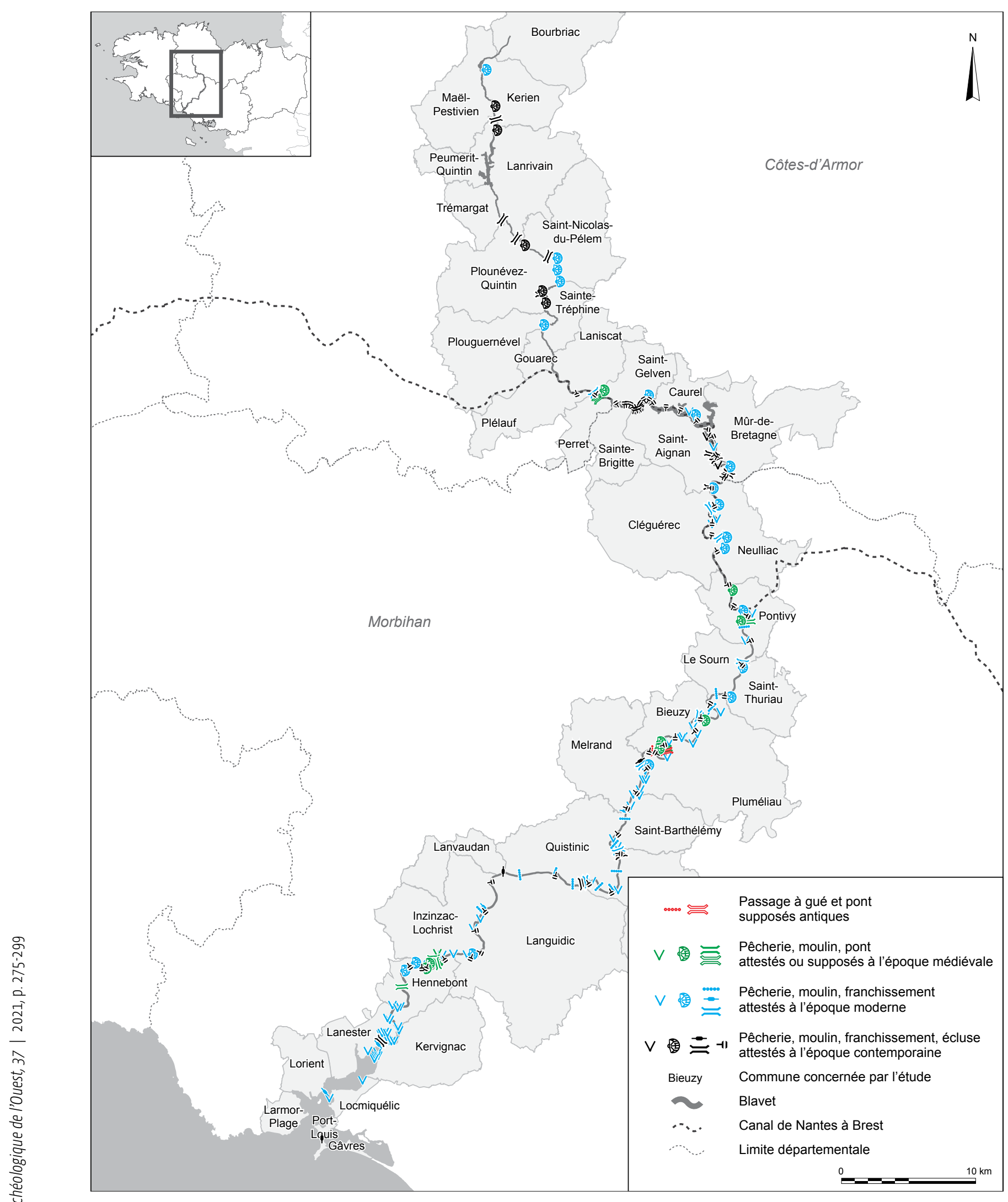

Figure 18 : Synthèse (réal. P. Peter, source : arch. dép. Morbihan).

Figure 18: Overview (O P. Peter, source: arch. dép. Morbihan). 
ments préexistants (Peter, 2015, p. 137, 139). La mise en place d'un suivi archéologique lors de l'assec temporairement des biefs du canal devrait être envisagée pour procéder au recensement de ces potentiels vestiges habituellement immergés.

La prise en considération des documents d'archives (mémoires de reconnaissances, plans dressés par des ingénieurs-géographes, etc.) apparaît comme essentielle car ces sources apportent des quantités d'informations, exploitables dans le cadre de telles études. Réalisés sur demande de l'administration et supervisés par des ingénieurs qualifiés, ces mémoires et reconnaissances sont relativement fiables et souvent très précis quant à la position et à l'architecture de ces aménagements fluviaux.

\section{CONCLUSION}

$\mathrm{Au}$ terme de cette première enquête sur le Blavet, près de deux cents sites fluviaux ont été répertoriés. Les plans dressés par les ingénieurs en charge de la canalisation du cours d'eau, accompagnés de leurs remarques, constituent une source d'information précieuse : leur qualité participe à la localisation relativement précise des aménagements supprimés lors de la construction du canal, dans le premier quart du XIx ${ }^{e} s$. Aujourd'hui détruits ou fortement détériorés, tous témoignent d'une occupation particulièrement dense de la rivière, au moins depuis le Moyen Âge. Au total, ce sont 62 pêcheries, 32 moulins, 13 passages à gué, 5 bacs, 22 ponts qui ont été identifiés sur le Blavet, de la source à l'estuaire, auxquels s'ajoutent quelques sites non localisés (fig. 18). Les écluses à sas, intrinsèquement liées à la canalisation du Blavet, ont elles aussi été comptabilisées. Au nombre de soixante, elles assurent le bon fonctionnement du canal. Aujourd'hui, seules vingt-huit d'entre elles sont encore actives : les trente-deux autres sont pour certaines désaffectées, et pour d'autres, immergées sous les eaux du lac de Guerlédan.

Plus largement, ce recensement a mis en lumière - à travers l'exemple du Blavet et de la documentation produite dans le cadre de sa canalisation - l'intérêt archéologique des canaux de navigation. Leur étude répond, en effet, à des problématiques développées en archéologie fluviale, mais aussi, dans le cas du Blavet, par une archéologie des périodes moderne et contemporaine. En complément de la poursuite des recherches en archives, plus spécifiquement orientées sur les pêcheries (enquête en cours de Jean-François Nicolas), le suivi de l'entretien des biefs, temporairement mis à sec, ne peut que compléter les informations réunies dans le cadre de cette enquête, qui met en lumière un cours d'eau intensément exploité et transformé depuis le Moyen Âge jusqu’à nos jours.

\section{Bibliographie}

ANDré P., 1992, "Un atelier de salaisons chez les Vénètes d'Armorique ", Revue des études anciennes, 94, 1-2, p. 41-47.

Barruol G., Fiches J.-L. et Garmy P. (dir.), 2011, Les ponts routiers en Gaule romaine, actes du colloque du Pont-du-Gard (8 au 11 octobre 2008), Montpellier, Presses universitaires de la Méditerranée, $41^{\mathrm{e}}$ supplément à la Revue archéologique de Narbonnaise, 720 p.

Benferhat K., Aubert S. et Nourry R., 1999, Le canal de Nantes à Brest, Rennes, Ouest-France, $142 \mathrm{p}$.

Billard C. et Bernard V. (dir.), 2016, Pêcheries de Normandie. Archéologie et histoire des pêcheries littorales du département de la Manche, Rennes, Presses universitaires de Rennes, 720 p.

Boudon J.-O., 2006, La France et l'Europe de Napoléon, Paris, Armand Colin, 352 p.

Canu B. et Billard C., 2016, "Anguilles et saumons : d'autres formes d'exploitation des ressources littorales ", dans BILLard C. et Bernard V. (dir.), Pêcheries de Normandie. Archéologie et histoire des pêcheries littorales du département de la Manche, Rennes, Presses universitaires de Rennes, p. 595-605.

Chaline O., 2004, La France au xviii siècle, Paris, Belin, 327 p.

Chasles F.-J., 1725, Dictionnaire universel, chronologique et historique de justice, police et finance, distribué par ordre de matière, contenant tous édits, déclarations du Roi, lettres patentes et arrests du conseil d'État, rendus depuis l'année 600 jusques et compris l'année 1720, Paris, imprimerie Claude Robustel, vol. 2, 796 p.

Cogné J., Horrenberger J.-C. et Morzadec M.-T., 1972, Carte géologique détaillée de la France à $1 / 50000$ : Lorient, 383, Orléans, Bureau de recherches géologiques et minières, $19 \mathrm{p}$.

Croix A. (dir.), 2006, La Bretagne d'après l'itinéraire de M. Dubuisson-Aubenay, Rennes, Presses universitaires de Rennes, $1118 \mathrm{p}$.

Driard C., Dréano Y. et Garnier N., 2017, " Les sauces de poisson produites sur la côte atlantique des Gaules : sources archéologiques et productions diversifiées des ateliers de salaison ", Gallia, 74-2, p. 183-205.

Duhamel du Monceau H.-L., 1769-1782, Traité général des pesches et histoire des poissons qu'elles fournissent, tant pour la subsistance des hommes, que pour plusieurs autres usages qui ont rapport aux Arts et au Commerce, Paris, Librairies Saillant \& Nyon et Desaint, 4 vol.

Dumont A., 2002, Les passages à gué de la Grande Saône : approche archéologique et historique d'un espace fluvial (de Verdun-surle-Doubs à Lyon), Dijon, Société archéologique de l'Est, $17^{\mathrm{e}}$ supplément à la Revue archéologique de l'Est, $280 \mathrm{p}$.

Dumont A., (dir.), 2006, Archéologie des lacs et des cours d'eau, Paris, Errance, 166 p. 
Dumont A., Foucher M., Steinmann L., Lavier C., Moyat P. et Garcia J.-P., 2014, « Évolution des ponts et $\mathrm{du}$ lit mineur de la Loire, entre La Charité-sur-Loire et La Chapelle-Montlinard ", Développement durable et territoires, 5-3, p. 1-16.

ÉveIllard J.-D., 1984, De l'incidence de la canalisation du Blavet et du relèvement de son plan d'eau sur les moulins et les minoteries entre Pontivy et Baud, mémoire de maitrise, Rennes, université de Rennes II, 157 p.

Fillon D., 2015, "Des pêcheries dans le fleuve à SaintFlorent-le-Vieil (Maine-et-Loire) : premier bilan ", dans Arthuis R. et Monteil M. (dir.), Archéologie de la BasseLoire, Rennes, Presses universitaires de Rennes, p. 107-140.

Galliou P. (dir.), 2009, Le Morbihan, Paris, Les Belles-Lettres, Carte archéologique de la Gaule, 56, 445 p.

Jaccottey L. et Rollier G. (dir.), 2016, Archéologie des moulins hydrauliques, à traction animale et à vent des origines à l'époque médiévale et moderne en Europe et dans le monde méditerranéen, actes du colloque international (Lons-le-Saunier, 2-5 novembre 2011), Besançon, Presses universitaires de Franche-Comté, 2 vol., 883 p.

Kersauson J. de, 1886, " Mémoires pour servir à l'histoire de la canalisation de la Bretagne ", Revue historique de l'Ouest, 2, p. 95-119.

Langouët L., Daire M.-Y. et Quesnel L., 2008, « Les pêcheries maritimes et estuariennes du Morbihan : première approche interdisciplinaire ", dans DAIRE M.-Y. et LANGouËT L. (dir.), Les pêcheries de Bretagne, archéologie et histoire des pêcheries d'estran, Rennes, Centre régional d'archéologie d'Alet et AMARAI, p. 123-143.

Le Mené J.-M., 1982, Histoire archéologique, féodale et religieuse des paroisses $d u$ diocèse de Vannes, Paris, Laffitte, vol. 2, 536 p. (1 ${ }^{\mathrm{re}}$ éd. : Vannes, Le Gall, 1891-1894).

Martin P., 2018, "Des havres, des passages et des pêcheries. Les petits aménagements estuariens en Bretagne sous l'Ancien Régime ", dans Llinares S., Égrasse B.et Dana K. (dir.), 2018, De l'estran à la digue. Histoire des aménagements portuaires et littoraux, $X V I^{e}-X X^{e}$ s., Rennes, Presses universitaires de Rennes, p. 309-318.
Mauret-Cribellier V. et Schemmama V., 2013, "Le cahier des données : les équipements du Cher liés à la navigation ", dans Serna V. (dir.), Le Cher. Histoire et archéologie d'un cours d'eau, Tours, FERACF, $43^{\mathrm{e}}$ supplément à la Revue archéologique du Centre de la France, p. 158-159.

NaAs P., 1999, Histoire rurale des Vénètes armoricains ( $V^{e} s$. av. J.C.-III s. apr. J.-C.), Saint-Malo, Centre régional d'archéologie d'Alet, $238 \mathrm{p}$.

Nicolas J.-F. et Cheyrouze D., 2015, Le Blavet et sa vallée, de Pontivy à Hennebont avant sa canalisation, Pays de Baud Histoire et Patrimoine, hors-série, 9, 99 p.

Nicolas J.-F. et Cheyrouze D., 2019, La canalisation du Blavet, de Pontivy à Hennebont et ses conséquences. 1800-1900, Pays de Baud Histoire et Patrimoine, hors-série, 13, 164 p.

Peter P., 2015, Bilan des connaissances sur le Blavet : une étude documentaire au service de l'archéologie fluviale et préventive, mémoire de master 2, université de Nantes, 194 p.

Phelypeaux L., 1726, Lettres patentes du Roy qui commettent le Sieur Le Masson Du Parc Commissaire de la Marine, pour faire l'inspections des pesches du poisson de mer sur les costes de Flandre, Picardie, Normandie \& Bretagne, Paris, Imprimerie royale, p. 1-3.

Pinon P., 1986, Un canal, des canaux, catalogue de l'exposition présentée à la Conciergerie du 7 mars au 8 juin 1986, Paris, Picard, $415 \mathrm{p}$.

Pinon P., 2005, Patrimoine fluvial, canaux et rivières navigables, Paris, SCALA, 255 p.

Serna V. (dir.) 2013, Le Cher. Histoire et archéologie d'un cours d'eau, Tours, FERACF, $43^{\mathrm{e}}$ supplément à la Revue archéologique du Centre de la France, 326 p.

Violet P.-L., 2004, L'hydraulique dans les civilisations anciennes. 5000 ans d'histoire, Paris, Presses de l'École nationale des ponts et chaussées, $383 \mathrm{p}$.

Wouters I. et Wibaut R., 2019, "Structures en fonte, fer, acier. Les enjeux de leurs commandes ", dans Bienvenu G., Monteil M. et Rousteau-Chambon H. (dir.), Construire! Entre Antiquité et Époque moderne, actes du $3^{e}$ congrès francophone d'histoire de la construction organisé à Nantes, 21-23 juin 2017, Paris, Picard, p. 581-590. 


\section{Vom Fluss zum Kanal: Untersuchung zum kulturgeschichtlichen Erbe des Flusses Blavet (Bretagne)}

Der Blavet ist ein Fluss der Bretagne, der im Laufe des 19. Jahrhunderts etwa zur Hälfte kanalisiert wurde. Die Auswertung von Dokumenten der französischen Straßen- und Wasserstraßenverwaltung hat es ermöglicht, zahlreiche Einrichtungen entlang des Wasserlaufs (Fischfanganlagen, Mühlen, Furte, etc.) zu dokumentieren, die damals bei umfangreichen Begradigungs- und Ausbauarbeiten teilweise zerstört wurden. Durch diese in ihrer Qualität einmalige Dokumentation konnten nicht nur Informationen zu diesen Anlagen gewonnen werden, sondern sie gaben auch einen Einblick in die Entscheidungen der damaligen Ingenieure, die damals den Fluss ausbauten und begradigten.

Schlagwortindex: Bretagne, Blavet, Schriftquellen, kulturgeschichtliches Erbe, Mittelalter.

\section{Del río al canal: investigaciones sobre el patrimonio fluvial del río Blavet (Bretaña) desde la Edad Media hasta nuestros días}

El Blavet es un rio bretón cuyo curso fue en parte canalizado durante el siglo XIX. El estudio de los archivos de los servicios de gestión de aguas ha permitido estudiar numerosas obras hidráulicas (pesquerias, molinos, vados, etc.) parcialmente destruidas durante su canalización a gran escala. Esta documentación de calidad excepcional aporta también información sobre la arquitectura de esas instalaciones, así como sobre las decisiones tomadas por los ingenieros para canalizar el río.

Palabras clave: Bretaña, Blavet, archivos, patrimonio fluvial, Edad Media. 
